\title{
Pacific
}

Journal of

Mathematics

\section{STABILITY OF THE KÄHLER-RICCI FLOW IN THE SPACE OF KÄHLER METRICS}

\author{
KAI ZHENG
}




\title{
STABILITY OF THE KÄHLER-RICCI FLOW IN THE SPACE OF KÄHLER METRICS
}

\author{
KAI ZHENG
}

\begin{abstract}
We prove that on a Fano manifold $M$ admitting a Kähler-Ricci soliton $(\omega, X)$, if the initial Kähler metric $\omega_{\varphi_{0}}$ is close to $\omega$ in a certain weak sense, then the weak Kähler-Ricci flow exists globally and converges in the sense of Cheeger and Gromov. In particular, $\varphi_{0}$ is not assumed to be $K_{X}$-invariant. The methods used are based on the metric geometry of the space of the Kähler metrics and are potentially applicable to other stability problems of geometric flows near the corresponding critical metrics.
\end{abstract}

\section{Introduction}

The Ricci flow was first introduced by Hamilton [1982] and now plays an important role in understanding the geometric and topological structure of the manifolds it lives on. We call the Ricci flow a Kähler-Ricci flow if the underlying manifold is a Kähler manifold. The normalized Kähler-Ricci flow is given by

$$
\left\{\begin{array}{l}
\frac{\partial}{\partial t} \omega=-\operatorname{Ric}+\lambda \omega, \\
\omega(0)=\omega_{\varphi_{0}},
\end{array}\right.
$$

where $\omega(0)$ stays in the canonical class $2 \pi C_{1}(M)$ and $\lambda$ is the sign of the first Chern class. Cao [1985] first showed that the Kähler-Ricci flow (1-1) has longtime existence. He also proved that the Kähler-Ricci flow converges to a KählerEinstein metric when the first Chern class is negative or zero. Now we restrict ourselves to the case where the first Chern class is positive. Since the KählerRicci flow preserves the Kähler class, we can rewrite the Kähler-Ricci flow in terms of the Ricci potential:

$$
\left\{\begin{array}{l}
\frac{\partial \varphi}{\partial t}=\log \frac{\omega_{\varphi}^{n}}{\omega^{n}}+\varphi-h_{\omega}+a(t), \\
\varphi(0)=\varphi_{0}
\end{array}\right.
$$

where $a$ is a function of $t$ and the Ricci potential $h_{\omega}$ of the reference metric $\omega$ is

MSC2000: primary 32Q20, 53C25; secondary 53C55, 58E11.

Keywords: Kähler-Ricci flow, space of Kähler metrics, stability. 
defined by

$$
\sqrt{-1} \partial \bar{\partial} h_{\omega}=\operatorname{Ric}(\omega)-\omega \quad \text { and } \quad \int_{M} e^{h_{\omega}} \omega^{n}=\operatorname{Vol}(M) .
$$

The convergence of the Kähler-Ricci flow has been studied by many authors. Chen and Tian [2002; 2006] proved it for Kähler-Einstein manifolds under the assumption of positivity of the Ricci curvature along the flow. Perelman (unpublished; see detailed proof in [Sesum and Tian 2008]) obtained an estimate of the Kähler-Ricci flow and proved that it converges to a Kähler-Einstein metric in the sense of Cheeger-Gromov, when one exists for any initial Kähler metric. Tian and Zhu [2007] extended this to the case of a Kähler-Ricci soliton for a $K_{X}$-invariant initial metric; a Kähler-Ricci soliton is a Kähler metric such that there is a holomorphic vector field $X$ satisfying

$$
L_{X} \omega=\operatorname{Ric}-\omega .
$$

Since the right side of (1-4) is real-valued, we obtain $L_{\operatorname{Im} X} \omega=0$ and $\operatorname{Im} X$, the imaginary part of $X$, generates a one-parameter isometry group $K_{X}$. Without assuming that $M$ admits a Kähler-Einstein metric or a Kähler-Ricci soliton, the analytic and geometric conditions of the convergence of the Kähler-Ricci flow are studied in [Phong and Sturm 2006; Phong et al. 2009, 2008; 2011, Tosatti 2010; Székelyhidi 2010; Munteanu 2009; Pali 2009; Chen and Wang 2010; Rubinstein 2009.

In order to study the asymptotic behavior of the Kähler-Ricci flow, we consider the flow's stability problem. That is, on a Kähler manifold $M$ admitting a KählerRicci soliton $(\omega, X)$, for what kind of neighborhood of $\omega$ does the Kähler-Ricci flow with initial datum in that neighborhood converge (in some sense - maybe exponentially) to the Kähler-Ricci soliton?

This stability problem has been investigated by many people; for references, see [Chen and Li 2009]. That work and [Tian and Zhu 2008] consider perturbing both the initial metric and the complex structure near a Kähler-Einstein metric.

In this paper, we focus on perturbing the initial metric near the Kähler-Ricci soliton without changing the complex structure. The main results of this paper are as follows, where $\mathcal{N}\left(\epsilon_{0} ; B, p\right)$ is a small neighborhood of the zero function, to be specified in Section 6.

First we will give a direct proof, based on the geometry of the space of Kähler metrics, of long-time existence and convergence in the Cheeger-Gromov sense, within the frame of Donaldson's program [2004]. The result is this:

Theorem 1.1. If a Kähler manifold admits a Kähler-Ricci soliton $(\omega, X)$, there exists a positive constant $\epsilon_{0}$ such that, if the initial potential $\varphi_{0}$ stays in $\mathcal{N}\left(\epsilon_{0} ; B, p\right)$, the weak Kähler-Ricci flow exists globally and converges in the Cheeger-Gromov 
sense. If , moreover, $\varphi_{0}$ is $K_{X}$-invariant, the weak modified Kähler-Ricci flow converges exponentially to a unique Kähler-Ricci soliton nearby.

When the Futaki invariant vanishes, it is obvious that the holomorphic vector fields $X$ is zero and the Kähler-Ricci soliton is a Kähler-Einstein metric.

Theorem 1.2. On a Kähler-Einstein manifold, there exists a positive constant $\epsilon_{0}$ such that, if the initial potential $\varphi_{0}$ stays in $\mathcal{N}\left(\epsilon_{0} ; B, p\right)$, the weak Kähler-Ricciflow exists globally and converges exponentially to a unique Kähler-Einstein metric nearby.

Simon [1983] studied the asymptotic behavior of the gradient flow of the variation problem via the Łojasiewicz-Simon inequality, which compares the distance to the critical set with the norm of the gradient of the functional in the $L^{2}$ space under the condition that the functional should be analytic. The underlying idea is to reduce the infinite-dimensional problem to a finite-dimensional problem. Perelman [2002] introduced a new functional, called the $\mu$ functional, and pointed out that the Ricci flow is the gradient flow of the $\mu$ functional up to a diffeomorphism.

We will not apply the Łojasiewicz-Simon inequality to the $\mu$ functional directly. Instead, we provide a new approach to the study of the asymptotic behavior of the flow which is merely a pseudogradient flow of some functional, since in the Kähler setting, geometry gives us more information. To be precise, the critical set in the space of Kähler metrics is a finite-dimensional Riemannian symmetric space, which we will explain later.

Since the Kähler-Ricci flow is the pseudogradient flow of the $K$-energy, in order to make the mechanism of our proof more clear, we first prove Theorem 1.2 under the assumption that the $C^{2, \alpha}$ norm of $\varphi_{0}$ is small. Then we generalize our approach to the case of a Kähler-Ricci soliton (Theorem 1.1).

A sketch of the proofs goes as follows. We first prove that the Kähler-Ricci flow (1-2), after pullback by the corresponding holomorphic transformations, will always stay in a small neighborhood of the background Kähler-Einstein metric. When $M$ has no nontrivial holomorphic vector field, it is not necessary to find the transformations; Section 3 gives a proof of this. However, in general, when $M$ admits nontrivial holomorphic vector fields, we need a new method, developed in Section 4A, to pick up the appropriate transformations following the trace of the Kähler-Ricci flow in the space of normalized Kähler potential $\mathscr{H}_{0}$; see (2-2). It has been shown by Mabuchi [1987], Donaldson [1999] and Semmes [1992] independently that $\mathscr{H}_{0}$ is an infinite-dimensional symmetry space of negative curvature. Later, Chen [2000b] proved $\mathscr{H}_{0}$ is also a metric space. Since the space $\mathscr{E}_{0}$ of potentials of Kähler-Einstein metrics is a totally geodesic submanifold in $\mathscr{H}_{0}$, the projection $\rho$ minimizing the distance function from the Kähler-Ricci flow to $\mathscr{E}_{0}$ is uniquely determined. The Bando-Mabuchi uniqueness theorem [1987] on 
the Kähler-Einstein metric implies that $\omega_{\rho}$ is different from the reference KählerEinstein metric by a holomorphic transformation. The projection Kähler-Einstein metric is exactly the new reference metric we've acquired.

Another way to derive a holomorphic transformation (Section 7) of $\varphi \in \mathscr{H}_{0}$ is to minimize in $\mathscr{E}_{0}$ the $I-J$ functional, introduced in [Bando and Mabuchi 1987] to prove the uniqueness of the Kähler-Einstein metric. However, this method cannot be applied in our case directly, since in general the hessian of the $I-J$ functional is not strictly positive; that is, the minimizer is not unique. Nevertheless, as we observed when the $C^{2, \alpha}$ norm of $\varphi$ is small, the hessian of the $I-J$ functional is indeed strictly positive. Therefore, the holomorphic transformation is uniquely determined.

In Section 5, we prove stability of the Kähler-Ricci flow near a Kähler-Ricci soliton $(\omega, X)$, similarly to the case of Kähler-Einstein metric. We use $(\omega, X)$ as the background metric. We first prove that the Kähler-Ricci flow (1-2) with small $C^{2, \alpha}$ initial Kähler potential will always stay in a small neighborhood of $\omega$ in the Cheeger-Gromov sense. The key idea is to use Perelman's $\mu$ functional [2002] instead of the $K$-energy, since the hessian of the $\mu$ functional is nonnegative at a Kähler-Ricci soliton within the canonical class [Tian and Zhu 2008]. Furthermore, we reparametrize the Kähler-Ricci flow (1-1) by the automorphisms $\varsigma(t)$ generated by the real part $\operatorname{Re} X$ of $X$ such that

$$
\left\{\begin{array}{l}
\frac{\partial}{\partial t} \omega_{\phi}=-\operatorname{Ric}\left(\omega_{\phi}\right)+\omega_{\phi}+L_{\operatorname{Re} X} \omega_{\phi}, \\
\omega_{\phi(0)}=\omega_{\varphi_{0}} .
\end{array}\right.
$$

It is obvious that the Kähler-Ricci soliton is the stationary solution of the modified Kähler-Ricci flow (1-5). Since the Kähler-Ricci soliton $(\omega, X)$ is $K_{X}$-invariant and the Kähler-Ricci flow is also invariant under the holomorphic diffeomorphism, we assume without loss of generality that the initial datum is $K_{X}$-invariant. Then the exponential convergence of the modified Kähler-Ricci flow follows from [Phong et al. 2011].

Finally, in Section 6, at a fixed time, we show that the $C^{2, \alpha}$ norm of the potential is small when the initial value is small under certain weak conditions. The main idea is to use the estimate introduced in [Chen et al. 2008].

As a corollary of Theorem 1.1, we deduce that the limit metric of the KählerRicci flow is unique. Let $\left\{\varphi\left(t_{i}\right)\right\}$ be a sequence of solutions of the Kähler-Ricci flow converging to a Kähler-Einstein metric or Kähler-Ricci soliton $g_{\infty}$, if one exists; then there exists some $\varphi \in\left\{\varphi\left(t_{i}\right)\right\}$ satisfying the stability condition of Theorem 1.1. According to that condition, the Kähler-Ricci flow with initial value $\varphi$ converges exponentially to a Kähler-Einstein metric $g_{\infty}^{1}$ or Kähler-Ricci soliton, respectively. Further, since we assume that $\left\{\varphi\left(t_{i}\right)\right\} \rightarrow g_{\infty}$, we must have $g_{\infty}^{1}=g_{\infty}$. 
We stress that the approach used to prove Theorem 1.1 is also applicable to the case of the general pseudogradient flow: neither the condition that the flow is the gradient flow of some functional, nor Perelman's deep estimate, nor a prior longtime existence of the flow is required. It is possible that our method can be used to solve other, similar problems of geometric flow, such as the stability of the pseudoCalabi flow near a constant scalar curvature Kähler metric in [Chen and Zheng 2010] and of the Calabi flow near a extremal metric in [Huang and Zheng 2010].

The paper is organized as follows: in Section 2 we review known results on the space of Kähler metrics and the well-posedness of the pseudo-Calabi flow obtained in [Chen and Zheng 2010] — see (2-10). In Sections 3 and 4 we prove Theorem 1.2 under the assumption that the $C^{2, \alpha}$ norm of the initial Kähler potential is small. Then we prove Theorem 1.1 under the same assumption in Section 5. Finally, in Section 6 we explain how to weaken the initial condition to the one stated in Theorem 1.2 and Theorem 1.1. In Section 7, we explain another method to choose the holomorphic transformation.

\section{Notation and basic results}

Let $M$ be a compact Kähler manifold of complex dimension $n$ with positive first Chern class $C_{1}(M)$ and let $\omega$ be a Kähler form representing the canonical class $2 \pi C_{1}(M)$. In a local holomorphic coordinate $z_{1}, z_{2}, \ldots, z_{n}$, the form $\omega$ is expressed by

$$
\omega=\sqrt{-1} \sum_{i=1}^{n} g_{i \bar{j}} d z^{i} \wedge d z^{\bar{j}}
$$

The corresponding Riemannian metric is given by

$$
g=\sum_{i=1}^{n} g_{i} \bar{j} d z^{i} \otimes d z^{\bar{j}} .
$$

For a Kähler metric $\omega$, the volume form is

$$
d V=\omega^{n}=(\sqrt{-1})^{n} \operatorname{det}\left(g_{i \bar{j}}\right) d z^{1} \wedge d z^{\overline{1}} \wedge \cdots \wedge d z^{n} \wedge d z^{\bar{n}} .
$$

The Ricci form

$$
\text { Ric }=\sqrt{-1} \sum_{i=1}^{n} R_{i} \bar{j} d z^{i} \wedge d z^{\bar{j}}=-\sqrt{-1} \partial \bar{\partial} \log \operatorname{det} \omega^{n}
$$

is a closed real $(1,1)$-form and belongs to $2 \pi C_{1}(M)$. Accordingly, the scalar curvature satisfies

$$
S \omega^{n}=n \operatorname{Ric} \wedge \omega^{n-1} .
$$

A direct calculation gives the average scalar curvature:

$$
\underline{S}=\frac{1}{V} \int_{M} S d V=\frac{n}{V} \int_{M} \operatorname{Ric} \wedge \omega^{n-1}=n .
$$


Let $\mathscr{K}$ be the set of Kähler forms on $M$ representing $2 \pi C_{1}(M)$ and let $\mathscr{E}$ be the set of Kähler-Einstein metrics in $\mathscr{K}$. According to the $\partial \bar{\partial}$ lemma, for any Kähler metric $\omega^{\prime}$ in $\mathscr{K}$ there exists a smooth real-valued function $\varphi$ such that $\omega^{\prime}=\omega+\sqrt{-1} \partial \bar{\partial} \varphi$. Then the space of Kähler potentials of $\mathscr{K}$ is given by

$$
\mathscr{H}=\left\{\varphi \in C^{\infty}(M, \mathbb{R}) \mid \omega+\sqrt{-1} \partial \bar{\partial} \varphi \in \mathscr{K}\right\} .
$$

Apparently, we have an isomorphism

$$
T \mathscr{H} \cong \mathscr{H} \times C^{\infty}(M, \mathbb{R}) .
$$

Mabuchi [1987], Donaldson [1999] and Semmes [1992] independently defined a Riemannian metric on $\mathscr{H}$ by

$$
\int_{M} f_{1} f_{2} \omega_{\varphi}^{n}
$$

for any $f_{1}, f_{2} \in T_{\varphi} \mathscr{H}$. For any path $\varphi(t)(0 \leq t \leq 1)$ in $\mathscr{H}$, the length is given by

$$
L(\varphi(t))=\int_{0}^{1} \sqrt{\int_{M} \varphi^{\prime}(t)^{2} \omega_{\varphi(t)}^{n}} d t,
$$

and the geodesic equation is

$$
\varphi^{\prime \prime}(t)-\frac{1}{2}\left|\nabla_{t} \varphi^{\prime}(t)\right|_{\varphi(t)}^{2}=0,
$$

where ' denotes differentiation in $t$ and $\nabla_{t}$ denotes the covariant derivative for the metric $g_{\varphi(t)}$. The geodesic equation enables us to define the connection on the tangent bundle. For any tangent vector field $\psi(t)$ along the path $\varphi(t)$, the covariant derivative along $\varphi(t)$ is defined by

$$
D_{t} \psi=\frac{\partial \psi}{\partial t}-\frac{1}{2}\left(\nabla_{t} \psi, \nabla_{t} \varphi^{\prime}\right)_{g_{\varphi}} .
$$

Then the connection at $\varphi$ is given by

$$
G(X \mid Y)\left(\psi_{1}, \psi_{2}\right)=-\frac{1}{2}\left(\nabla \psi_{1}, \nabla \psi_{2}\right)_{g_{\varphi}},
$$

for any $\psi_{1}$ and $\psi_{2}$ in $T_{\varphi} \mathscr{H} . G(X \mid Y)$ is torsion-free and metric-compatible.

Theorem 2.1 [Mabuchi 1987; Donaldson 1999; Semmes 1992]. The Riemannian manifold $\mathscr{H}$ is an infinite-dimensional symmetric space; it admits a Levi-Civita connection whose curvature is covariant constant. At a point $\varphi \in \mathscr{H}$ the curvature is given by

$$
R_{\varphi}\left(\delta_{1} \varphi, \delta_{2} \varphi\right) \delta_{3} \varphi=-\frac{1}{4}\left\{\left\{\delta_{1} \varphi, \delta_{2} \varphi\right\}_{\varphi}, \delta_{3} \varphi\right\}_{\varphi},
$$

where $\{,\}_{\varphi}$ is the Poisson bracket on $C^{\infty}(M)$ of the symplectic form $\omega_{\varphi}$. 
Theorem 2.2 [Chen 2000b]. He is a metric space, and is convex by $C^{1,1}$ geodesics.

Calabi and Chen [2002] proved $\mathscr{H}$ is negatively curved in the sense of Alexandroff. We denote the space of normalized Kähler potentials by

$$
\mathscr{H}_{0}=\left\{\varphi \in C^{\infty}(M, R) \mid \omega+\sqrt{-1} \partial \bar{\partial} \varphi>0 \text { and } I(\varphi)=0\right\},
$$

where

$$
I(\varphi)=\frac{1}{V} \sum_{p=0}^{n} \frac{1}{(p+1) !(n-p) !} \int_{M} \varphi \omega^{n-p} \wedge(\partial \bar{\partial} \varphi)^{p} .
$$

In fact, $\mathscr{H}$ can be naturally split as

$$
\mathscr{H}=\mathscr{H}_{0} \times \mathbb{R} .
$$

This leads to a decomposition of the tangent space:

$$
T_{\varphi}=\left\{f \mid \int_{M} f \omega_{\varphi}^{n}=0\right\} \oplus \mathbb{R} .
$$

On a Kähler-Einstein manifold $(M, \omega)$, choose $\omega$ be the reference metric. It is clear from the definition (1-3) that $h_{\omega}=0$. Substituting this into the potential equation (1-2) of the Kähler-Ricci flow, we obtain

$$
\left\{\begin{array}{l}
\frac{\partial \varphi}{\partial t}=\log \frac{\omega_{\varphi}^{n}}{\omega^{n}}+\varphi+a(t), \\
\varphi(0)=\varphi_{0} .
\end{array}\right.
$$

If we choose the normalization constant in (2-3) appropriately, namely,

$$
a(t)=-\frac{1}{V} \int_{M}\left(\log \frac{\omega_{\varphi}^{n}}{\omega^{n}}+\varphi\right) \omega_{\varphi}^{n}
$$

we see that

$$
\partial_{t} I(\varphi)=\frac{1}{V} \int_{M} \partial_{t} \varphi \omega_{\varphi}^{n}=0 .
$$

We first assume that $\varphi_{0} \in \mathscr{H}_{0}$ satisfies $I\left(\varphi_{0}\right)=0$; the general case will be treated in Section 6. Then (2-5) implies $I(\varphi)=0$, which ensures that the solution $\varphi$ of (2-3) always stays in $\mathscr{H}_{0}$.

For any $\varphi \in \mathscr{H}$, Mabuchi [1986] defined the $K$-energy of $(M, \omega)$ as

$$
\nu\left(\omega, \omega_{\varphi}\right)=-\frac{1}{V} \int_{0}^{1} \int_{M} \dot{\varphi}(\tau)\left(S_{\varphi(\tau)}-\underline{S}\right) \omega_{\varphi(\tau)}^{n} d \tau,
$$


where $\varphi(\tau)$ is an arbitrary piecewise smooth path from 0 to $\varphi$. An explicit expression of the $K$-energy is formulated in [Chen 2000a; Tian 2000] as

$$
\begin{aligned}
v_{\omega}(\varphi)=\frac{1}{V} \int_{M} \log \frac{\omega_{\varphi}^{n}}{\omega^{n}} \omega_{\varphi}^{n}+\frac{\underline{S} n !}{V} I(\varphi) \\
\quad-\frac{1}{V} \sum_{i=0}^{n-1} \frac{n !}{(i+1) !(n-i-1) !} \int_{M} \varphi \operatorname{Ric} \wedge \omega^{n-1-i} \wedge(\partial \bar{\partial} \varphi)^{i} .
\end{aligned}
$$

In later sections we will simply write $v(\varphi)$ instead of $v_{\omega}(\varphi)$.

Theorem 2.3 [Mabuchi 1987]. If $\omega$ is a critical point of $v(\varphi)$, the second variation of the K-energy satisfies

$$
\left.\frac{d^{2}}{d t^{2}} v\left(\theta_{t}\right)\right|_{t=0} \geq 0
$$

for every smooth path $\left\{\theta_{t} \mid-\epsilon \leq t \leq \epsilon\right\}$ in $\mathscr{K}$ such $\theta_{0}=\omega$.

Let $\operatorname{Aut}(M)$ be the group of holomorphic automorphisms of $M$ and $\operatorname{Aut}_{0}(M)$ its identity component.

Theorem 2.4 [Bando and Mabuchi 1987; Bando 1987]. Assume $\mathscr{E} \neq \varnothing$.

(i) The K-energy is bounded from below on $\mathscr{K}$ and takes its absolute minimum exactly on $\mathscr{E}$.

(ii) $\mathscr{E}$ consists a single $\operatorname{Aut}_{0}(M)$-orbit.

Indeed, the normalization constant $a(t)$ can be estimated by the $K$-energy.

Lemma 2.5. Let $\varphi$ be the solution of (2-3). The relation between $a(t)$ and the $K$-energy $v(\varphi)$ is given by

$$
a(t)+v(\varphi)=a(0)+v\left(\varphi_{0}\right) .
$$

Proof. We calculate the evolution of $a(t)$ along the Kähler-Ricci flow directly:

$$
V \frac{d}{d t} a(t)=-\int_{M}\left(\triangle_{\varphi}+1\right) \dot{\varphi} \omega_{\varphi}^{n}-\int_{M}\left(\log \frac{\omega_{\varphi}^{n}}{\omega^{n}}+\varphi\right) \triangle_{\varphi} \dot{\varphi} \omega_{\varphi}^{n} .
$$

By Stokes' theorem and (2-5) the first term vanishes identically. Integration by parts and the use of (2-3) gives for the second term

$$
\int_{M}\left(S_{\varphi}-n\right) \dot{\varphi} \omega_{\varphi}^{n}
$$

Since (2-6) implies

$$
\frac{d}{d t} v(t)=-\frac{1}{V} \int_{M}\left(S_{\varphi}-n\right) \dot{\varphi} \omega_{\varphi}^{n},
$$

we obtain $\frac{d}{d t} a(t)=-\frac{d}{d t} v(t)$. We conclude by integrating both sides with respect to $t$. 
Since the $K$-energy is decreasing along the Kähler-Ricci flow, we immediately conclude the following according to Theorem 2.4.

Corollary 2.6. On a Kähler-Einstein manifold, a(t) is uniformly bounded along the Kähler-Ricci flow.

Set

$$
X=C^{0}\left([0, T), C^{2+\alpha}(M, g)\right) \cap C^{1}\left([0, T), C^{\alpha}(M, g)\right) .
$$

The following theorems asserting short-time existence, regularity and continuous dependence on initial data for the Kähler-Ricci flow were proved by Chen and the author, who defined a new second-order Monge-Ampère flow, called the pseudoCalabi flow and coinciding with the Kähler-Ricci flow when the initial datum is restricted in the canonical Kähler class:

$$
\left\{\begin{array}{l}
\frac{\partial \varphi}{\partial t}=-f(\varphi), \\
\triangle_{\varphi} f(\varphi)=S(\varphi)-\underline{S} .
\end{array}\right.
$$

Theorem 2.7 [Chen and Zheng 2010]. Let $\varphi_{0} \in C^{2, \alpha}(M, g)$ be such that

$$
\lambda \omega \leq \omega_{\varphi_{0}} \leq \Lambda \omega,
$$

for two positive constants $\lambda$ and $\Lambda$. Then the pseudo-Calabi flow has a unique solution $\varphi(x, t) \in X$, where $T$ is the maximal existence time.

Theorem 2.8 [Chen and Zheng 2010]. The solution of the pseudo-Calabi flow $\varphi \in X$ is smooth for any $t>0$. More precisely, if $|\varphi(t)|_{C^{2, \alpha}} \leq A$ for any $0 \leq t \leq T$, there exists a constant $C$ (depending on $A, g, t_{0}$ and $k$ ) such that $|\varphi(t)|_{C^{k, \alpha}} \leq C$ for any $T-t_{0} \leq t \leq t_{0}<T$.

Theorem 2.9 [Chen and Zheng 2010]. If $\phi$ is the solution of the pseudo-Calabi flow for an initial datum $\phi_{0}$ on $[0, T]$, there is a neighborhood $U$ of $\phi_{0}$ such that the pseudo-Calabi flow has a solution $\varphi(t)$ on $[0, T]$ for any $\varphi_{0} \in U$ and the mapping $\varphi_{0} \mapsto \varphi(t)$ is $C^{k}$ for $k=0,1,2, \ldots$

As a corollary of the continuous dependence on initial data we have:

Theorem 2.10 [Chen and Zheng 2010]. Suppose $M$ admits a constant scalar curvature Kähler metric $\omega$. Let $\varphi_{0} \in C^{2, \alpha}(M, g)$ be such that $\lambda \omega \leq \omega_{\varphi_{0}} \leq \Lambda \omega$ for positive constants $\lambda$ and $\Lambda$. Then for any $T>0$ there exits a positive constant $\epsilon_{0}(T)$ such that, if $\left|\varphi_{0}\right|_{C^{2, \alpha}(M, g)} \leq \epsilon_{0}(T)$, the pseudo-Calabi flow has a unique solution on $[0, T]$ and

$$
|\dot{\varphi}|_{C^{\alpha}(M, g)}+|\varphi|_{C^{2, \alpha}(M, g)} \leq C \epsilon_{0}(T) \quad \text { for all } t \in[0, T],
$$

where $C$ depends on $M, g$ and $T$. As $T$ goes to infinity, $\epsilon_{0}(T)$ goes to zero. 


\section{No nontrivial holomorphic vector fields}

Let $\eta(M)$ be the set of all holomorphic vector fields on $M$. We start with the case $\eta(M)=\varnothing$. We shall prove the following proposition in this section.

Proposition 3.1 [Tian and Zhu 2008; Zhu 2009]. Assume M admits a KählerEinstein metric $\omega$ and has no holomorphic vector fields. There exits a small positive constant $\epsilon_{0}$ such that, if the initial datum satisfies

$$
\left|\varphi_{0}\right|_{C^{2, \alpha}(M)} \leq \epsilon_{0},
$$

then the Kähler-Ricci flow $g_{\varphi}$ converges smoothly to $g$.

Proof. We at first show that under the assumption of the proposition, the solution of (2-3) always stays in some small $\epsilon_{1}$-neighborhood of the zero function.

Lemma 3.2. For any $\epsilon_{1}>0$, there exits a small positive constant $\epsilon_{0}$ such that, if $\left|\varphi_{0}\right|_{C^{2, \alpha}(M)} \leq \epsilon_{0}$, then $|\varphi(t)|_{2, \alpha} \leq \epsilon_{1}$ for all $t \in[0,+\infty)$.

Proof. Suppose that the conclusion fails; then there exists a sequence of initial data $\varphi_{s}^{0}$ such that

$$
\left|\varphi_{s}^{0}\right|_{C^{2, \alpha}} \leq \frac{1}{s}
$$

By virtue of Theorem 2.10, we get a sequence of solutions $\varphi_{s}(t)$ satisfying the flow equations (2-3) with $\varphi_{s}(0)=\varphi_{s}^{0}$. Let $T_{s}$ be the first time such that

$$
\left|\varphi_{s}\left(T_{s}\right)\right|_{C^{2, \alpha}}=\epsilon_{1} \quad \text { and } \quad\left|\varphi_{s}(t)\right|_{C^{2, \alpha}}<\epsilon_{1} \text { for } 0 \leq t<T_{s} .
$$

According to Theorem 2.10 again, we have $T_{s} \geq T_{1}>0$. Moreover, we apply Theorem 2.8 to (2-3) on $\left[T_{s}-2 a, T_{s}\right]$ for fixed $a$ such that $0<a<T_{s} / 2-T_{1} / 4$, then we obtain a uniform higher-order bound for the sequence of solutions:

$$
\left|\varphi_{s}\right|_{C^{k, \alpha}(M)} \leq C\left(k, \epsilon_{1}, a\right) \quad \text { on }\left[T_{s}-a, T_{s}\right], \text { for all } k \geq 0 .
$$

Consequently, there is a subsequence of $\phi_{s}=\varphi_{s}\left(T_{s}\right)$ converges smoothly to $\phi_{\infty}$ satisfying

$$
\left|\phi_{\infty}\right|_{C^{2, \alpha}}=\epsilon_{1} .
$$

It is obvious that $g_{\phi_{\infty}}$ is still a Kähler metric. Since the $K$-energy is not only well defined for $\varphi_{s}^{0}$ by (2-7) but also decreasing along the Kähler-Ricci flow, Theorem 2.4 implies that

$$
0 \leq v_{\omega}\left(\phi_{s}\right) \leq v_{\omega}\left(\varphi_{s}(0)\right) \leq \frac{C}{s} .
$$

By passing the limit we obtain

$$
\lim _{s \rightarrow \infty} v_{\omega}\left(\varphi_{s}\right)=v_{\omega}\left(\varphi_{\infty}\right)=0
$$


Using Theorem 2.4, we obtain that $g_{\phi_{\infty}}$ is a Kähler-Einstein metric. From the same theorem we deduce that $\phi_{\infty}$ must be a constant. Furthermore the normalization condition $I\left(\phi_{\infty}\right)=0$ leads to $\phi_{\infty}=0$, which contradicts to (3-2). The lemma follows.

According to Theorem 2.8 and Lemma 3.2, we have $|\varphi(t)|_{C^{k}} \leq C_{k}$ for any $k \geq 3$ away from $t=0$. It follows that for any sequence $t_{i}$ there is a subsequence such that $\phi\left(t_{i}\right)$ converges smoothly to a limit function $\varphi_{\infty}$. Moreover, since the $K$-energy has a lower bound and it decays along the flow, $\omega_{\varphi_{\infty}}$ must be a Kähler-Einstein metric. This, together with Theorem 2.4 and the normalization condition, implies that $\varphi_{\infty}=0$. Because the $t_{i}$ can be chosen arbitrarily, we conclude that the KählerRicci flow converges smoothly to the original Kähler-Einstein metric.

\section{4. $M$ admits nontrivial holomorphic vector fields}

4A. Choice and estimate of holomorphic transformations. When $M$ admits holomorphic vector fields, we need to find an appropriate holomorphic transformation. Let $\mathscr{E}_{0} \subset \mathscr{H}_{0}$ be the space of Kähler potentials of Kähler-Einstein metrics.

Let $\sigma_{t}^{*} \omega$ be any curve with $\sigma_{0}=\mathrm{id}$ in $\mathscr{E}_{0}$. The tangent vector at $\omega$ is

$$
\left.\frac{d}{d t} \sigma_{t}^{*}\right|_{t=0} \omega=L_{X} \omega \text {. }
$$

Here $X=\left.\left(\sigma_{t}\right)_{*}^{-1} \partial_{t} \sigma_{t}\right|_{t=0}$ is the real part of some holomorphic vector field. Since $C_{1}(M)>0$ implies that $M$ is simply connected by [Kobayashi 1961], we obtain $L_{X} \omega=\sqrt{-1} \partial \bar{\partial} \theta_{X}$ for some function $\theta_{X}$. Hence, the finite-dimensionalness of the space of holomorphic vector fields implies that of $\mathscr{E}_{0}$. Moreover, according to [Mabuchi 1987], $\mathscr{E}_{0}$ is also a totally geodesic submanifold of $\mathscr{H}_{0}$. Then the point $\rho \in \mathscr{E}_{0}$ realizes the shortest distance between $\varphi$, and $\mathscr{E}_{0}$ is uniquely determined. In fact, according to Theorem 2.4, we obtain a holomorphic diffeomorphism $\sigma \in \operatorname{Aut}_{0}(M)$ such that $\sigma^{*} \omega=\omega+\sqrt{-1} \partial \bar{\partial} \rho$. The $K$-energy is invariant under holomorphic transformations:

Lemma 4.1 [Mabuchi 1986]. $v\left(\omega, \omega_{\left(\sigma^{-1}\right)^{*}(\varphi-\rho)}\right)=v\left(\omega, \omega_{\varphi}\right)=v\left(\omega_{\rho}, \omega_{\varphi}\right)$.

Proof. Since $\omega$ and $\omega_{\rho}$ are both Kähler-Einstein metrics, Lemma (5.4.1) and Theorem (5.3) of [Mabuchi 1986] yield, respectively, the equalities

$$
\begin{aligned}
v\left(\omega, \omega_{\left(\sigma^{-1}\right)^{*}(\varphi-\rho)}\right) & =v\left(\sigma^{*} \omega, \omega_{\varphi}\right)=v\left(\omega_{\rho}, \omega_{\varphi}\right) \\
& =v\left(\omega_{\rho}, \omega\right)+v\left(\omega, \omega_{\varphi}\right)=v\left(\omega, \omega_{\varphi}\right) .
\end{aligned}
$$

We next state two lemmas from [Chen and Zheng 2010] regarding the metric rephrased for economy

geometry of the space of constant scalar curvature Kähler metrics. They show that when metrics stay close to $\omega$, their projection metrics are uniformly bounded. 
Lemma 4.2 [Chen and Zheng 2010]. There exists a positive constant $\epsilon$ such that $|\rho|_{C^{3, \alpha}} \leq C_{2} \epsilon$ for any $\rho$ satisfying $d(0, \rho) \leq \epsilon$.

Proof. Since $\mathscr{E}_{0}$ is a finite-dimensional Riemannian symmetric space, a small $\epsilon$ neighborhood near $\rho=0$ in this submanifold can be pulled back by the exponential map $\exp _{0}$ to the tangent space $T_{0}\left(\mathscr{E}_{0}\right)$ at 0 . Set $\psi=\exp _{0}^{-1}(\rho)$. Then the length from $\psi$ to 0 is $\epsilon$. The norm induced by the distance on $T_{0}\left(\mathscr{E}_{0}\right)$ is equivalent to the $C^{2, \alpha}$ norm, since all norms on a finite-dimensional vector space are equivalent. Thus $\left|\exp _{0}^{-1}(\rho)\right|_{C^{2, \alpha}}$ is bounded by $C_{1} \epsilon$. Since the exponential map is a diffeomorphism in the $\epsilon$ neighborhood near $\rho=0$, we obtain $|\rho|_{C^{2, \alpha}} \leq C_{2} \epsilon$ for some constant $C_{2}$. The lemma follows by an appropriate choice of $\epsilon$.

We can improve this conclusion for $C^{k}$ for fixed $k \geq 0$, not only for $C^{3, \alpha}$ norm.

Lemma 4.3 [Chen and Zheng 2010]. There exists a positive constant $\epsilon_{1}$ such that $|\varphi|_{C^{2, \alpha}} \leq \epsilon_{1}$ implies

$$
|\rho|_{C^{3, \alpha}} \leq C_{4} \text { and }|\sigma|_{h} \leq C_{5},
$$

where $h$ is the left invariant metric in $\operatorname{Aut}(M)$.

Proof. Choose a path $\gamma_{t}=t \varphi-I(t \varphi) \in \mathscr{H}_{0}$ for $0 \leq t \leq 1$. Denote $d(0, \varphi)$ the distance between 0 and $\varphi$. Using (2-1), we compute

$$
\begin{aligned}
d(0, \varphi) \leq L\left(\gamma_{t}\right) & =\int_{0}^{1}\left(\int_{M}\left(\frac{\partial \gamma_{t}}{\partial t}\right)^{2} \omega_{\gamma_{t}}^{n}\right)^{1 / 2} d t \\
& =\int_{0}^{1}\left(\int_{M}\left(\varphi-\partial_{t} I(t \varphi)\right)^{2} \omega_{\gamma_{t}}^{n}\right)^{1 / 2} d t \leq C_{3} \epsilon_{1}
\end{aligned}
$$

for $|\varphi|_{C^{2, \alpha}} \leq \epsilon_{1}$. Moreover, the choice of the $\rho$ implies

$$
d(0, \rho) \leq d(0, \varphi)+d(\varphi, \rho) \leq 2 d(0, \varphi) \leq C_{3} \epsilon_{1},
$$

by the triangle inequality. From Lemma 4.2, it follows that $|\rho|_{C^{3, \alpha}} \leq C_{4}=C_{2} C_{3} \epsilon_{1}$. Using [Chen and Tian 2006, Lemma 4.6], we derive $|\sigma|_{h} \leq C_{5}$ and the lemma follows.

Remark. Alternatively the holomorphic transformation can be derived by minimizing the $I-J$ functional, as in [Bando and Mabuchi 1987], which will be further discussed in Section 7. Those authors use this minimizer to prove the uniqueness of the Kähler-Einstein metric when the first Chern class is positive. The minimizer of the $I-J$ functional is not unique in general, since the second variation of this functional is not strictly positive. However, when the potential is small enough, the minimizer is unique. We mention also that Corollary 7.2 provides an estimate similar to Lemma 4.3. 
4B. Long time existence and Cheeger-Gromov convergence. Set

$$
\mathscr{S}\left(\epsilon_{1}, C\left(k, \epsilon_{1}\right)\right)=\left\{\varphi|\varphi|_{C^{2, \alpha}} \leq \epsilon_{1} ;|\varphi|_{C^{k, \alpha}(M)} \leq C\left(k, \epsilon_{1}\right)\right\} .
$$

It is obvious that $0 \in \mathscr{Y}$. We will show that when the initial potential is small, the solution of (2-3) always stays in $\mathscr{Y}$ after pulling back by a sequence of holomorphic transformations.

Lemma 4.4. For any $\epsilon>0$, there is a small positive constant $o$ depends on $\epsilon$ and $\mathscr{Y}$ such that, for any $\varphi \in \mathscr{Y}$, if $v_{\omega}(\varphi) \leq o$, then $\left|\left(\sigma^{-1}\right)^{*}(\varphi-\rho)\right|_{C^{2, \alpha}}<\epsilon$.

Proof. If the conclusion fails, we take a positive constant $\epsilon$ and a sequence of $\varphi_{s} \in \mathscr{Y}$ satisfying

$$
v_{\omega}\left(\varphi_{s}\right) \leq \frac{1}{s}
$$

and such that

$$
\left|\left(\sigma_{s}^{-1}\right)^{*}\left(\varphi_{s}-\rho_{s}\right)\right|_{C^{2, \alpha}} \geq \epsilon .
$$

Since $\varphi_{s} \in \mathscr{Y}\left(\epsilon_{1}, C\left(k, \epsilon_{1}\right)\right)$, we obtain a subsequence $\varphi_{s_{j}}$ of $\varphi_{s}$ converging smoothly to $\varphi_{\infty}$. Let $\hat{\varphi}_{s}=\left(\sigma_{s}^{-1}\right)^{*}\left(\varphi_{s}-\rho_{s}\right)$. Lemma 4.3 gives

$$
\left|\rho_{s}\right|_{C^{3, \alpha}} \leq C_{4} \quad \text { and } \quad\left|\sigma_{s}\right|_{h} \leq C_{5},
$$

which implies that there are, by the Arzelà-Ascoli theorem and the BolzanoWeierstrass theorem respectively, subsequences of $\rho_{s_{j}}$ and $\sigma_{s_{j}}$ for which (using the same notation)

and

$$
\rho_{s_{j}} \rightarrow \rho_{\infty} \text { in } C^{3, \beta} \quad \text { for any } \beta<\alpha
$$

$$
\sigma_{s_{j}} \rightarrow \sigma_{\infty} \text { in the left invariant metric. }
$$

Combining with Lemma 4.1, which implies that

$$
v_{\omega}\left(\varphi_{\infty}\right)=v_{\omega}\left(\hat{\varphi}_{\infty}\right)=0,
$$

we derive that $\hat{\varphi}_{s_{j}}$ converges to $\hat{\varphi}_{\infty}=\left(\sigma_{\infty}^{-1}\right)^{*}\left(\varphi_{\infty}-\rho_{\infty}\right) \in \mathscr{E}_{0}$ in $C^{3, \beta}$ and $\sigma_{\infty}^{*} \omega=$ $\omega+\partial \bar{\partial} \rho_{\infty}$. Moreover, according to Theorem 2.4, we have $\hat{\varphi}_{\infty}, \varphi_{\infty} \in \mathscr{E}_{0}$.

We claim that

$$
d\left(\varphi_{\infty}, \rho_{\infty}\right)=0 .
$$

Otherwise, for some sufficient large $N$, when $s_{j}>N$,

$$
d\left(\varphi_{s_{j}}, \rho_{s_{j}}\right)=d\left(\varphi_{s_{j}}, \mathscr{E}_{0}\right)
$$

has a strictly positive lower bound. Since the distance function is at least $C^{1}$ (see [Chen 2000b]), we have $d\left(\varphi_{\infty}, \mathscr{E}_{0}\right)>0$, contradicting $\varphi_{\infty} \in \mathscr{E}_{0}$. Consequently, the claim holds. It follows that $\hat{\varphi}_{\infty}=0$, in contradiction with the lower bound $\left|\hat{\varphi}_{\infty}\right|_{C^{2, \alpha}} \geq \epsilon$ of (4-1). 
Proposition 4.5. Assume $M$ admits a Kähler-Einstein metric $\omega$ and has nontrivial holomorphic vector fields. There is a small positive constant $\epsilon_{0}$ such that, if $\left|\varphi_{0}\right|_{C^{2, \alpha}(M)} \leq \epsilon_{0}$, there is a unique solution $\varphi(t)$ and a corresponding holomorphic transformation $\varrho(t)$ such that the normalization potential of $\varrho(t)^{*} \omega(t)$ always stays in $\mathscr{Y}$. Moreover, any sequence $t_{j}$ has is a subsequence (still denoted by $t_{j}$ ) such that $\varrho\left(t_{j}\right)^{*} \omega\left(t_{j}\right)$ converges smoothly to a Kähler-Einstein metric $\omega_{\infty}$.

Proof. We prove this proposition by contradiction. Let $\epsilon_{1}$ be as in Lemma 4.3. Using Theorem 2.10, we assume there is a maximal time $T$ such that

$$
|\varphi|_{C^{2, \alpha}}<\epsilon_{1} \text { on }[0, T) \quad \text { and } \quad|\varphi(T)|_{C^{2, \alpha}}=\epsilon_{1} .
$$

According to Theorem 2.8 we obtain $|\varphi(T)|_{C^{k, \alpha}} \leq C\left(k, \epsilon_{1}, t_{0}, g\right)$ on $\left[T-t_{0}, T\right]$ for a fixed $T / 2 \leq t_{0} \leq T$. Let the constant $C\left(k, \epsilon_{1}\right)$ be $C\left(k, \epsilon_{1}, t_{0}, g\right)$. So we get

$$
\varphi(T) \in \mathscr{S}\left(\epsilon_{1}, C\left(k, \epsilon_{1}\right)\right) .
$$

There are two situations. If $\varphi(T)$ is a Kähler-Einstein metric, the flow will stop here and our theorem is proved. Otherwise, we will extend the flow as follows.

We first choose $\epsilon_{0}$ small enough to guarantee

$$
v_{\omega}\left(\varphi_{0}\right) \leq o\left(\frac{\epsilon_{1}}{2}, \mathscr{Y}\left(\epsilon_{1}, C\left(k, \epsilon_{1}\right)\right)\right),
$$

where the constant $o\left(\epsilon_{1} / 2, \mathscr{Y}\left(\epsilon_{1}, C\left(k, \epsilon_{1}\right)\right)\right)$ is determined in Lemma 4.4. Let the holomorphic transformation $\sigma$ be the projection of $\varphi(T)$ in $\mathscr{E}_{0}$ with

$$
\sigma^{*} \omega=\omega+\sqrt{-1} \partial \bar{\partial} \rho .
$$

We set $\varphi_{1}^{0}$ be the Kähler potential of the metric pulled back by $\sigma$, that is,

$$
\left(\sigma^{-1}\right)^{*} \omega_{\varphi(T)}=\omega+\sqrt{-1} \partial \bar{\partial}\left[\left(\sigma^{-1}\right)^{*}(\varphi(T)-\rho)\right]=\omega+\sqrt{-1} \partial \bar{\partial} \varphi_{1}^{0} .
$$

Since the $K$-energy decreases along the Kähler-Ricci flow, Lemma 4.1 yields

$$
v_{\omega}\left(\varphi_{1}^{0}\right) \leq o\left(\frac{\epsilon_{1}}{2}, \mathscr{Y}\left(\epsilon_{1}, C\left(k, \epsilon_{1}\right)\right)\right) .
$$

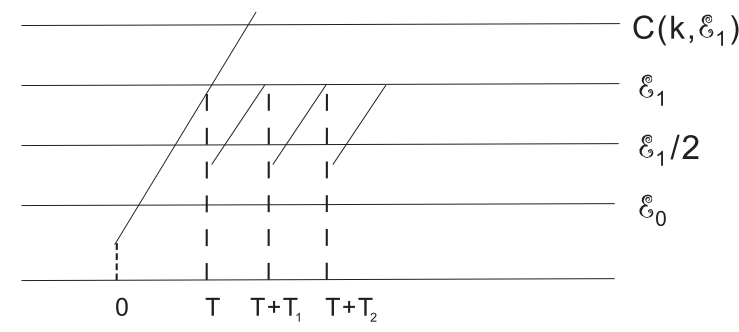

Idea of the proof of Proposition 4.5: solving the equation after pulling back. 
Letting $\psi=\left(\sigma^{-1}\right)^{*}(\varphi(T)-\rho)$, Lemma 4.4 implies that

$$
|\psi|_{C^{2, \alpha}(g)}=\left|\left(\sigma^{-1}\right)^{*}(\varphi(T)-\rho)\right|_{C^{2, \alpha}(g)}<\frac{\epsilon_{1}}{2} .
$$

We next show that the Kähler-Ricci flow is invariant under the transformation. Let $\varphi_{1}=\left(\sigma^{-1}\right)^{*}(\varphi(t)-\rho)$. We compute

$$
\begin{aligned}
\frac{\partial}{\partial t} \varphi_{1} & =\left(\sigma^{-1}\right)^{*}\left[\log \frac{\omega_{\varphi}^{n}}{\omega^{n}}+\varphi-\frac{1}{V} \int_{M}\left(\log \frac{\omega_{\varphi}^{n}}{\omega^{n}}+\varphi\right) \omega_{\varphi}^{n}\right] \\
& =\left(\sigma^{-1}\right)^{*}\left[\log \frac{\omega_{\varphi}^{n}}{\omega_{\rho}^{n}}+\varphi-\rho-\frac{1}{V} \int_{M}\left(\log \frac{\omega_{\varphi}^{n}}{\omega_{\rho}^{n}}+\varphi-\rho\right) \omega_{\varphi}^{n}\right] \\
& =\left[\log \frac{\omega_{\varphi_{1}}^{n}}{\omega^{n}}+\varphi_{1}-\frac{1}{V} \int_{M}\left(\log \frac{\omega_{\varphi_{1}}^{n}}{\omega^{n}}+\varphi_{1}\right) \omega_{\varphi_{1}}^{n}\right] .
\end{aligned}
$$

The second equality follows form the fact that $\omega_{\rho}$ is a Kähler-Einstein metric. We conclude that $\varphi_{1}$ is the solution of an equation of the form

$$
\left\{\begin{array}{l}
\frac{\partial}{\partial t} \varphi_{1}=\log \frac{\omega_{\varphi_{1}}^{n}}{\omega^{n}}+\varphi_{1}+a(t) \\
\varphi_{1}(0)=\varphi_{1}^{0}=\left(\sigma^{-1}\right)^{*}(\varphi(T)-\rho),
\end{array}\right.
$$

where (4-3) and (4-2) hold. Again, Theorem 2.10 implies (4-4) has a solution on $\left[0, T_{1}\right]$ with $T_{1} \geq T$ such that

$$
\left|\varphi_{1}\left(T_{1}\right)\right|_{C^{2, \alpha}}=\epsilon_{1} .
$$

According to Theorem 2.8, we also obtain

$$
\left|\varphi\left(T_{1}\right)\right|_{C^{k, \alpha}} \leq C\left(k, \epsilon_{1}, t_{0}, g\right) \quad \text { on }\left[T_{1}-t_{0}, T_{1}\right] .
$$

So we still have $\varphi\left(T_{1}\right) \in \mathscr{Y}\left(\epsilon_{1}, C\left(k, \epsilon_{1}\right)\right)$. Moreover, if we let

$$
\varphi(t)=\sigma^{*} \varphi_{1}(t-T)+\rho \quad \text { on }\left[T, T+T_{1}\right),
$$

the new $\varphi(t)$ is the solution of (2-3) on $\left[0, T+T_{1}\right]$.

We repeat the same steps inductively for

$$
\varphi_{s-1}\left(T_{s-1}\right) \in \mathscr{S}\left(\epsilon_{1}, C\left(k, \epsilon_{1}\right)\right),
$$

with $T_{s-1} \geq T$ obtained in Theorem 2.10, until $\varphi_{s}$ becomes a Kähler-Einstein at time $T_{s}$, with $T_{s}<\infty$. If this does not happen, the Kähler-Ricci flow has long-time existence and the solution $\varphi(t)$ for all $t \geq 0$ is given by

$$
\omega_{\varphi(t)}=\prod_{i=0}^{s-1} \sigma_{i}^{*} \omega_{\varphi_{s}(t)} \quad \text { on }\left[\sum_{i=0}^{s-1} T_{i}, \sum_{i=0}^{s} T_{i}\right) .
$$


Finally, we prove the convergence of the Kähler-Ricci flow. For any sequence $\left\{\varphi_{t_{j}}\right\}$, there is $s$ such that $\sum_{i=0}^{s-1} T_{i} \leq t_{j} \leq \sum_{i=0}^{s} T_{i}$. Let

We have

$$
\varrho_{j}=\left(\prod_{i=0}^{s-1} \sigma_{i}\right)^{-1} .
$$

$$
\left|\varrho_{j}^{*} \omega_{\varphi_{t_{j}}}-\omega\right|_{C^{\alpha}} \leq \epsilon_{1} \quad \text { and } \quad\left|\varrho_{j}^{*} \omega_{\varphi_{t_{j}}}-\omega\right|_{C^{k}} \leq C\left(k, \epsilon_{1}\right) .
$$

Therefore all metrics are equivalent and their derivatives are bounded. We set

$$
\omega_{\psi_{t_{j}}}=\varrho_{j}^{*} \omega_{\varphi_{t_{j}}} .
$$

It follows that there is a subsequence of $\omega_{\psi_{t_{j}}}$ that converges to a limit metric $\omega_{\infty}$ (which depends on the choice of the subsequence). Since the $K$-energy is bounded below, we have $\lim _{s \rightarrow \infty} v\left(\omega, \omega_{\psi_{j}}\right)=0$. It follows from Theorem 2.4 that $g_{\infty}$ is a Kähler-Einstein metric. The proposition is proved.

Let $t_{s}=\sum_{i=0}^{s} T_{i}$. Following the argument in [Chen and Tian 2006], we can first connect each pair of points $\varphi_{t_{s}}$ and $\varphi_{t_{s+1}}$ by a geodesic in the space of KählerEinstein metrics, so

$$
\varrho(t)=\varrho(s) \exp \left((t-s) X_{s}\right) \quad \text { for all } t \in[s, s+1],
$$

with $X_{s}$ uniformly bounded by Lemma 4.3. We then smooth the corner at each point $t_{s}$ by replacing the broken line by a smooth curve in a small neighborhood of $t_{s}$ without changing the value and the $t$ derivative at the endpoints. Hence we have extended the holomorphic transformation to all $t$, while ensuring Lipschitz continuity in $t$.

Let $\omega_{\psi(t)}=\varrho(t)^{*} \omega_{\varphi(t)}$. We have already seen that the Kähler-Ricci flow converges to a Kähler-Einstein metric in Cheeger-Gromov sense; i.e., for any sequence $g\left(t_{i}\right)$, there is a subsequence $g\left(t_{i_{j}}\right)$ and a holomorphic transformation $\varrho\left(t_{i_{j}}\right)$ such that $\varrho\left(t_{i_{j}}\right)^{*} g\left(t_{i_{j}}\right)$ converges smoothly to a Kähler-Einstein metric $g_{\infty}$. So we have

$$
\lim _{t \rightarrow \infty} \operatorname{Ric}\left(g_{\psi_{t}}\right)-\omega_{\psi_{t}}=0,
$$

which leads to the convergence of the eigenvalue. To obtain the compactness of the sequence of holomorphic transformations $\varrho(t)$ and the exponential convergence of the Kähler-Ricci flow, we use an auxiliary result:

Theorem 4.6 [Phong et al. 2009, Theorem 2 and Remark (7)]. If the Kähler-Ricci flow converges to a Kähler-Einstein metric in Cheeger-Gromov sense. Then the Kähler-Ricci flow must converge exponentially to a unique Kähler-Einstein metric nearby. 


\section{Kähler-Ricci solitons}

In this section we generalize our argument to the Kähler-Ricci solitons. According to [Fujiki 1978], the identity part of holomorphic transformation group $\operatorname{Aut}_{0}(M)$ is meromorphically isomorphic to a linear algebraic group $L(M)$ and the quotient $\operatorname{Aut}_{0}(M) / L(M)$ is a complex torus. Futaki and Mabuchi [1995] used the Chevalley decomposition to $L(M)$ to obtain a semidirect decomposition

$$
\operatorname{Aut}_{0}(M)=\operatorname{Aut}_{r}(M) \ltimes R_{u} .
$$

Here $\operatorname{Aut}_{r}(M)$ is the reductive algebra group, which is the complexification of a maximal compact subgroup $K$, and $R_{u}$ is the unipotent radical of $\operatorname{Aut}_{0}(M)$. Let $\eta_{r}$ be the Lie algebra of $\operatorname{Aut}_{r}(M)$. Recall that a Kähler metric $\omega$ is called a KählerRicci soliton if there is a holomorphic vector field $X$ such that

$$
L_{X} \omega=\operatorname{Ric}-\omega .
$$

Tian and Zhu [2000] proved the uniqueness of Kähler-Ricci solitons for a fixed $X$ in the Lie algebra of $\operatorname{Aut}_{0}(M)$.

Theorem 5.1 [Tian and Zhu 2000]. If $(\omega, X)$ and $\left(\omega^{\prime}, X\right)$ are Kähler-Ricci solitons, there are holomorphic transformations $\sigma \in \operatorname{Aut}_{0}(M)$ and $\tau \in \operatorname{Aut}_{r}(M)$ such that $\sigma^{*} \omega=\tau^{*} \sigma^{*} \omega^{\prime}$ and $\sigma^{*} X \in \eta_{r}$.

Theorem 5.2 [Tian and Zhu 2002]. If $(\omega, X)$ and $\left(\omega^{\prime}, X^{\prime}\right)$ are two Kähler-Ricci solitons, then there is a holomorphic transformation group $\sigma \in \operatorname{Aut}_{0}(M)$ such that $\omega=\sigma^{*} \omega^{\prime}$ and $X=\sigma_{*}^{-1} X^{\prime}$.

Since $L_{\operatorname{Im} X} \omega=0, \operatorname{Im} X$ generates a one-parameter isometric group $K_{X}$. We further choose $K$ such that $K_{X} \subseteq K$. According to Proposition 2.1 of [Tian and Zhu 2002], $X$ lies in the center of $\eta_{r}$.

Now we fix a holomorphic vector field $X$. By the Hodge theory there is a real value function $\theta_{X}$ such that $L_{X} \omega=\sqrt{-1} \partial \bar{\partial} \theta_{X}$ with $\int_{M} e^{\theta_{X}} \omega^{n}=V$. Then the potential equation of the Kähler-Ricci flow (1-2) is

$$
\left\{\begin{array}{l}
\frac{\partial \varphi}{\partial t}=\log \frac{\omega_{\varphi}^{n}}{\omega^{n}}+\varphi-\theta_{X}+a(t) \\
\varphi(0)=\varphi_{0}
\end{array}\right.
$$

We choose

$$
a(t)=-\frac{1}{V} \int_{M}\left(\log \frac{\omega_{\varphi}^{n}}{\omega^{n}}+\varphi-\theta_{X}\right) \omega_{\varphi}^{n}
$$

moreover $I\left(\varphi_{0}\right)=0$, so the Kähler-Ricci flow stays in $\mathscr{H}_{0}$. 
Recall the $W$-functional of [Perelman 2002], defined by

$$
\mathscr{W}(g, f, \tau)=(4 \pi \tau)^{-n / 2} \int_{M}\left[\tau\left(|\nabla f|^{2}+S\right)+f-n\right] e^{-f} d V,
$$

and invariant under diffeomorphisms $\sigma$ and scaling $C$ :

$$
\mathcal{W}\left(C \sigma^{*} g, \sigma^{*} f, C \tau\right)=\mathscr{W}(g, f, \tau) .
$$

Recall also Perelman's $\mu$ functional, defined by

$$
\mu(g, \tau)=\inf \left\{\mathcal{W}(g, f, \tau) \mid(4 \pi \tau)^{-n / 2} \int_{M} e^{-f} d V=1\right\},
$$

and also invariant under diffeomorphism. Its minimum is achieved by some smooth function $f$ satisfying $\tau\left[\left(2 \triangle f-|\nabla f|^{2}\right)+S\right]+f-n=\mu(g, \tau)$. The first variation of $\mu(g, \tau)$ at $g_{i j}^{\prime}=v_{i j}$ for fixed $\tau$ is

$$
\mu^{\prime}\left(v_{i j}, \tau\right)=(4 \pi \tau)^{-n / 2} \int_{M}\left\{-\tau\left(v_{i j}, \operatorname{Ric}+D^{2} f-\frac{1}{2 \tau} g\right)\right\} e^{-f} d V_{g} .
$$

So the (shrinking) Kähler-Ricci soliton is the critical point of $\mu\left(g, \tau=\frac{1}{2}\right)$. The gradient flow of the $\mu$ functional equals to (1-1) with $\lambda=1$ up to a diffeomorphism generated by $\nabla f$. So the $\mu$ functional is nondecreasing along the Ricci flow. The second variation of this functional near a Kähler-Ricci soliton in the canonical class has been calculated:

Theorem 5.3 [Tian and Zhu 2008, Proposition 2.1]. We have

$$
\left.\frac{\partial^{2}}{d t^{2}} \mu(\omega+\sqrt{-1} \partial \bar{\partial} \varphi)\right|_{t=0} \leq 0,
$$

and equality holds if and only if $\dot{\varphi}(0)$ is the real part of the holomorphic potential of some holomorphic vector field.

So the only directions in which the Kähler-Ricci soliton $\omega$ in (5-4) vanishes are those tangent to the orbit of $\omega$ under the action of $\operatorname{Aut}_{0}(M)$. We thus obtain the following local property of the $\mu$ functional:

Lemma 5.4. A Kähler-Ricci soliton is a local maximum of $\mu(g)$ in $\mathscr{H}_{0}$.

Proof. Near a Kähler-Ricci soliton $g$, the tangent space $T_{\omega}\left(\mathscr{H}_{0}\right)$ splits as $\eta(M) \oplus N$, where $N$ is the orthonormal part. Due to Theorem 5.3, $\mu\left(g^{\prime}\right)<\mu(g)$ along any direction in $N$. Moreover, since $\sigma^{*} g$ is still a Kähler-Ricci soliton for any $\sigma \in$ Aut $_{0}(m)$, we have $\mu\left(g^{\prime}\right) \equiv \mu(g)$ along any direction in $\eta(M)$.

As a result we deduce that a Kähler metric that achieves the maximum value of the $\mu(g)$ functional near a Kähler-Ricci soliton must be a Kähler-Ricci soliton.

Let $\mathscr{E}_{0} \subset \mathscr{H}_{0}$ be the space of potentials of Kähler-Einstein solitons with respect to the holomorphic vector field $X$. Due to Theorem $5.1, \mathscr{E}_{0}$ is a single orbit under the action of $\operatorname{Aut}_{r}(M)$. 
Lemma 5.5. $\mathscr{E}_{0}$ is a finite-dimensional totally geodesic submanifold of $\mathscr{H}_{0}$.

Proof. Analogously to the case of the extremal metric in [Calabi 1985], Lemma A.2 and Theorem A of [Tian and Zhu 2000] imply that the identity component of the holomorphic isometric group of the Kähler-Ricci soliton $(\omega, X)$ is a maximal compact subgroup of $\operatorname{Aut}_{r}(M)$ containing $K_{X}$. So $\left(\operatorname{Aut}_{r}(M), K\right)$ is a Riemannian symmetric pair and $\mathscr{E}_{0}$ is $\operatorname{Aut}_{r}(M)$-equivariantly diffeomorphic to the Riemannian symmetric space $\operatorname{Aut}_{r}(M) / K$. Then for any $\omega \in \mathscr{E}_{0}$, each geodesic starting at $\omega$ in $\mathscr{E}_{0}$ can be written in the form

$$
\gamma(t)=\exp (t \operatorname{Re} Y)^{*} \omega
$$

for some nonzero $Y$ whose imagine part is a Killing vector field. Then Theorem 3.5 and Remark 3.3 in [Mabuchi 1987] show that $\gamma(t)$ is also a geodesic in $\mathscr{H}_{0}$.

Now choose $\omega_{\rho}=\omega+\partial \bar{\partial} \rho$ such that $\rho$ realizes the shortest distance between $\psi$ and $\mathscr{E}_{0}$. Clearly, $\rho$ is uniquely determined. In fact, due to Theorem 5.1 we obtain a holomorphic diffeomorphism $\sigma \in \operatorname{Aut}_{r}(M)$ such that

$$
\sigma^{*} \omega=\omega_{\rho}=\omega+\sqrt{-1} \partial \bar{\partial} \rho,
$$

with $\rho \in \mathscr{E}_{0}$. By an argument analogous to the one in Proposition 4.5, but using the $\mu$ functional instead of the $K$-energy, we obtain:

Lemma 5.6. For any $\epsilon>0$, There is a small positive constant $o$ depends on $\epsilon$ and $\mathscr{Y}$ such that for any $\varphi \in \mathscr{Y}$, if $\mu\left(\omega_{\varphi}\right) \geq \mu(\omega)-o$, then $\left|\left(\sigma^{-1}\right)^{*}(\varphi-\rho)\right|_{C^{2, \alpha}}<\epsilon$.

Proof. If the conclusion fails, take a positive $\epsilon$ and a sequence of $\varphi_{s} \in \mathscr{Y}$ satisfying

$$
\mu\left(\omega_{\varphi_{s}}\right) \geq \mu(\omega)-\frac{1}{s} \text { and }\left|\left(\sigma_{s}^{-1}\right)^{*}\left(\varphi_{s}-\rho_{s}\right)\right|_{C^{2, \alpha}} \geq \epsilon .
$$

Since $\varphi_{s} \in \mathscr{S}$, we obtain a subsequence $\varphi_{s_{j}}$ of $\varphi_{s}$ converging smoothly to $\varphi_{\infty}$. Lemma 4.3 gives

$$
\left|\rho_{s}\right|_{C^{3, \alpha}} \leq C_{4} \quad \text { and } \quad\left|\sigma_{s}\right|_{h} \leq C_{5},
$$

which implies that $\left(\sigma_{s_{j}}^{-1}\right)^{*}\left(\varphi_{s_{j}}-\rho_{s_{j}}\right)$ converges in $C^{3, \beta}$ towards

$$
\hat{\varphi}_{\infty}=\left(\sigma_{\infty}^{-1}\right)^{*}\left(\varphi_{\infty}-\rho_{\infty}\right) \in \mathscr{E}_{0}, \quad \text { with } \sigma_{\infty}^{*} \omega=\omega+\partial \bar{\partial} \rho_{\infty} .
$$

Then (5-3) implies that $\mu\left(\omega_{\varphi_{\infty}}\right)=\mu\left(\omega_{\hat{\varphi}_{\infty}}\right)=\mu(\omega)$. The rest of this proof is the same as for Lemma 4.4 .

Proposition 5.7. Assume $M$ admits a Kähler-Ricci soliton $(\omega, X)$. There exits a small constant $\epsilon_{0}$ such that, if $\left|\varphi_{0}\right|_{C^{2, \alpha}(M)} \leq \epsilon_{0}$, there is a unique solution $\varphi(t)$ and a corresponding holomorphic transformation $\varrho(t) \in \operatorname{Aut}_{r}(M)$ such that the normalization potential of $\varrho(t)^{*} \omega_{\varphi}(t)$ always stays in $\mathscr{Y}$. Moreover, for any sequence $t_{i}$, there is a subsequence $t_{i_{j}}$ such that $\varrho\left(t_{i_{j}}\right)^{*} g_{\varphi\left(t_{i_{j}}\right)}$ converges smoothly to $g_{\infty}$. 
Proof. The proof, by contradiction, is similar to that of Proposition 4.5. Let $\epsilon_{1}$ be as in Lemma 4.3. Applying Theorem 2.10 to the potential equation (5-2), we assume there is a maximal time $T$ such that

$$
|\varphi|_{C^{2, \alpha}}<\epsilon_{1} \text { on }[0, T) \quad \text { and } \quad|\varphi(T)|_{C^{2, \alpha}}=\epsilon_{1} .
$$

From Theorem 2.8 we obtain $|\varphi(T)|_{C^{k, \alpha}} \leq C\left(k, \epsilon_{1}, t_{0}, g\right)$ on $\left[T-t_{0}, T\right]$ for a fixed $T / 2 \leq t_{0} \leq T$. Let the constant $C\left(k, \epsilon_{1}\right)$ be $C\left(k, \epsilon_{1}, t_{0}, g\right)$. So we get

$$
\varphi(T) \in \mathscr{S}\left(\epsilon_{1}, C\left(k, \epsilon_{1}\right)\right) .
$$

There are two situations. If $\varphi(T)$ is a Kähler-Ricci soliton, the flow will stop here and our theorem is proved. Otherwise, we will extend the flow as in the proof in Proposition 4.5.

We first choose $\epsilon_{0}$ small enough to guarantee that

$$
\mu\left(\omega_{\varphi_{0}}\right) \geq \mu(\omega)-o\left(\frac{\epsilon_{1}}{2}, \mathscr{Y}\left(\epsilon_{1}, C\left(k, \epsilon_{1}\right)\right)\right),
$$

where the constant $o\left(\epsilon_{1} / 2, \mathscr{Y}\left(\epsilon_{1}, C\left(k, \epsilon_{1}\right)\right)\right)$ is determined in Lemma 4.4. Let the holomorphic transformation $\sigma$ be the projection of $\varphi(T)$ in $\mathscr{E}_{0}$ with

$$
\sigma^{*} \omega=\omega+\sqrt{-1} \partial \bar{\partial} \rho .
$$

Let $\varphi_{1}^{0}$ be the Kähler potential of the metric pulled back by $\sigma$, that is,

$$
\left(\sigma^{-1}\right)^{*} \omega_{\varphi(T)}=\omega+\sqrt{-1} \partial \bar{\partial}\left[\left(\sigma^{-1}\right)^{*}(\varphi(T)-\rho)\right]=\omega+\sqrt{-1} \partial \bar{\partial} \varphi_{1}^{0} .
$$

Since the $\mu$ functional is nondecreasing along the Kähler-Ricci flow, we obtain

$$
\mu\left(\omega_{\varphi_{1}^{0}}\right) \geq \mu(\omega)-o\left(\frac{\epsilon_{1}}{2}, \mathscr{Y}\left(\epsilon_{1}, C\left(k, \epsilon_{1}\right)\right)\right) .
$$

Lemma 4.4 implies that

$$
\left|\left(\sigma^{-1}\right)^{*}(\varphi(T)-\rho)\right|_{C^{2, \alpha}(g)}<\frac{\epsilon_{1}}{2} .
$$

Set $\varphi_{1}(t)=\left(\sigma^{-1}\right)^{*}(\varphi(t)-\rho)$. Combining (5-2) and (5-1), we obtain that $\varphi_{1}$ is the solution of an equation of the form

$$
\left\{\begin{array}{l}
\frac{\partial}{\partial t} \varphi_{1}=\log \frac{\omega_{\varphi_{1}}^{n}}{\omega^{n}}+\varphi_{1}-\theta_{X}+a(t), \\
\varphi_{1}(0)=\varphi_{1}^{0}=\left(\sigma^{-1}\right)^{*}(\varphi(T)-\rho),
\end{array}\right.
$$

where (5-6) and (5-5) hold.

Again, Theorem 2.10 implies that (5-7) has a solution on [0, $\left.T_{1}\right]$, with $T_{1} \geq T$, such that

$$
\left|\varphi_{1}\left(T_{1}\right)\right|_{C^{2, \alpha}}=\epsilon_{1} .
$$


From Theorem 2.8, we also obtain $\left|\varphi\left(T_{1}\right)\right|_{C^{k, \alpha}} \leq C\left(k, \epsilon_{1}, t_{0}, g\right)$ on $\left[T_{1}-t_{0}, T_{1}\right]$. So we still have

$$
\varphi\left(T_{1}\right) \in \mathscr{Y}\left(\epsilon_{1}, C\left(k, \epsilon_{1}\right)\right) .
$$

If we set $\varphi(t)=\sigma^{*} \varphi_{1}(t-T)+\rho$ on $\left[T, T+T_{1}\right)$, the new $\varphi(t)$ is the solution of (5-2) on $\left[0, T+T_{1}\right]$.

We repeat the same steps inductively for

$$
\varphi_{s-1}\left(T_{s-1}\right) \in \mathscr{Y}\left(\epsilon_{1}, C\left(k, \epsilon_{1}\right)\right),
$$

with $T_{s-1} \geq T$ obtained in Theorem 2.10. We thus obtain a sequence of holomorphic transformations $\sigma_{i}$ and the solution $\varphi(t)$ for all $t \geq 0$ given by

$$
\omega_{\varphi(t)}=\prod_{i=0}^{s-1} \sigma_{i}^{*} \omega_{\varphi_{s}(t)} \quad \text { on }\left[\sum_{i=0}^{s-1} T_{i}, \sum_{i=0}^{s} T_{i}\right) .
$$

Set $\varrho_{j}=\left(\prod_{i=0}^{s-1} \sigma_{i}\right)^{-1}$. We have

$$
\left|\varrho_{j}^{*} \omega_{\varphi_{t_{j}}}-\omega\right|_{C^{\alpha}} \leq \epsilon_{1} \quad \text { and } \quad\left|\varrho_{j}^{*} \omega_{\varphi_{t_{j}}}-\omega\right|_{C^{k}} \leq C\left(k, \epsilon_{1}\right) .
$$

It follows that there is a subsequence of $\varrho_{j}^{*} \omega_{\varphi_{t_{j}}}$ converging to a limit metric $\omega_{\infty}$. According to Lemma 5.4, the $\mu$ functional is bounded above and $\omega_{\infty}$ is a KählerRicci soliton.

Assume $\varsigma$ is generated by $\operatorname{Re} X$ :

$$
\operatorname{Re} X=\left(\varsigma^{-1}\right)_{*} \frac{\partial}{\partial t} \varsigma
$$

Let $\varrho$ and $\phi$ satisfy $\varsigma^{*} \omega=\omega_{\varrho}$ and $\phi=\varsigma^{*} \varphi+\varrho$. We obtain the modified KählerRicci flow of the form

$$
\left\{\begin{array}{l}
\frac{\partial}{\partial t} \omega_{\phi}=-\operatorname{Ric}\left(\omega_{\phi}\right)+\omega_{\phi}+L_{\operatorname{Re} X} \omega_{\phi}, \\
\omega_{\varphi(0)}=\omega_{\varphi_{0}} .
\end{array}\right.
$$

We apply [Phong et al. 2011, Theorem 1] to obtain:

Theorem 5.8. If the Kähler-Ricci flow converges to a Kähler-Ricci soliton in the Cheeger-Gromov sense and the initial Kähler potential is $K_{X}$-invariant, then the modified Kähler-Ricci flow converges exponentially to a unique Kähler-Ricci soliton nearby.

Zhu [2009] also discussed the stability of Kähler-Ricci flow near a Kähler-Ricci soliton by using Perelman's estimate (unpublished) and Chen and Tian's energy method [2002; 2006]. 


\section{Weak flow}

In this section we weaken the initial condition. Let $a(t)=0$ in (2-3); the potential equation then reads

$$
\left\{\begin{array}{l}
\frac{\partial \varphi}{\partial t}=\log \frac{\omega_{\varphi}^{n}}{\omega^{n}}+\varphi, \\
\varphi(0)=\varphi_{0} .
\end{array}\right.
$$

We defined $\varphi_{0}$ is the limit of $\varphi_{s} \in \operatorname{PSH}(M, \omega) \cap L^{\infty}(M)$ in $L^{\infty}$ norm. Meanwhile, $\omega_{\varphi_{0}} \geq 0$ in the sense of currents. Let the weak solution be a limit of a sequence of approximation solutions by

$$
\varphi(t)=\lim _{s \rightarrow 0} \varphi(s, t) .
$$

The Kähler-Ricci flow with weak initial data was studied in [Chen and Ding 2007; Chen and Tian 2008; 2008]. We also have:

Theorem 6.1 [Song and Tian 2009, Proposition 3.2]. If $\varphi_{0}$ is defined above with $\left|\varphi_{0}\right|_{L^{\infty}} \leq A$ and $\left|\omega_{\varphi_{0}}^{n} / \omega^{n}\right|_{L^{p}(M, \omega)} \leq B$ for $p>1$, there is a unique smooth solution $g_{\varphi}(t)$ of (1-1) for $t>0$ such that

$$
\lim _{t \rightarrow 0^{+}} \varphi(t)=\varphi_{0} .
$$

The estimate in Song and Tian's proof is that

$$
|\varphi(t)|_{C^{k}} \leq C(t, T, k, A, B) \quad \text { on }(0, T] .
$$

For fixed $B$ and $p$, introduce the space

$$
\mathcal{N}\left(\epsilon_{0} ; B, p\right)=\left\{\varphi|\varphi|_{L^{\infty}} \leq \epsilon_{0},\left|\frac{\omega_{\varphi}^{n}}{\omega^{n}}\right|_{L^{p}(M, \omega)} \leq B \text { for some } p>1\right\},
$$

Here $B$ and $p$ should be chosen such that $\mathcal{N}\left(\epsilon_{0} ; B, p\right)$ is not the empty set. Clearly, if $\left|\varphi_{0}\right|_{C^{1,1}} \leq \epsilon_{0}$, then $\varphi_{0} \in \mathcal{N}\left(\epsilon_{0}, 1+\left(2^{n}-1\right) \epsilon_{0}, \infty\right)$. Actually, we have:

Lemma 6.2. Fix $t_{0} \in(0, T]$. For any $\epsilon_{1}>0$ there is a small $\epsilon_{0}$ such that for any $\varphi_{0} \in \mathcal{N}\left(\epsilon_{0} ; B, p\right)$ we have $\left|\varphi\left(t_{0}\right)\right|_{C^{2, \alpha}} \leq \epsilon_{1}$.

Proof. If the conclusion fails, choose a sequence of $\varphi_{s}$ such that

$$
\left|\varphi_{s}\right|_{L^{\infty}} \leq \frac{1}{s} \quad \text { and } \quad\left|\frac{\omega_{\varphi_{s}}^{n}}{\omega^{n}}\right|_{L^{p}(M, \omega)} \leq B .
$$

For each corresponding solution $\varphi_{s}(t)$ constructed by Theorem 6.1, we have

$$
\left|\varphi_{S}\left(t_{0}\right)\right|_{C^{2, \alpha}}>\epsilon_{1} .
$$


Setting $g_{a \varphi i \bar{j}}=\int_{0}^{t}\left(g_{i \bar{j}}+a \varphi_{i \bar{j}}\right) d a>0$, we rewrite (6-1) as follows

$$
\left\{\begin{array}{l}
\frac{\partial \varphi}{\partial t}=\triangle_{g_{a \varphi}} \varphi+\varphi, \\
\varphi_{s}(0)=\varphi_{s} .
\end{array}\right.
$$

From the maximum principle we obtain

$$
\sup _{M}\left|\varphi_{s}\left(t_{0}\right)\right| \leq e^{t_{0}} \sup _{M}\left|\varphi_{s}\right| \text {. }
$$

By (6-2), we can pass a subsequence of $\varphi_{s_{i}}\left(t_{0}\right)$ such that $\lim _{i \rightarrow \infty} \varphi_{s_{i}}\left(t_{0}\right)=\varphi_{\infty}\left(t_{0}\right)$ in $C^{k}$ for $k \geq 0$. Let $s=s_{i}$ in (6-4). Then the limit approaches $\sup _{M}\left|\varphi_{\infty}\left(t_{0}\right)\right| \leq 0$, which contradicts (6-3).

Now we have a $C^{2, \alpha}$ small initial datum $\varphi\left(t_{0}\right)$; we normalize it to be $\varphi_{0}-I\left(\varphi_{0}\right)$ which is also $C^{2, \alpha}$ small. Then we can solve Equation (2-3) with this initial datum. Combining Propositions 3.1 and 4.5, Theorem 4.6, and Lemma 6.2, we obtain Theorem 1.2. Analogously, we apply Proposition 5.7, Theorem 5.8 and Lemma 6.2 to obtain Theorem 1.1.

\section{Another choice of holomorphic transformations}

In this section, we follow the arguments in [Bando and Mabuchi 1987; Chen and Tian 2002] to find a good holomorphic transformation. The $I$ and $J$ functionals are defined as

$$
\begin{aligned}
& I\left(\omega, \omega_{\varphi}\right)=\frac{1}{V} \int_{M} \varphi\left(\omega^{n}-\omega_{\varphi}^{n}\right), \\
& J\left(\omega, \omega_{\varphi}\right)=\frac{1}{V} \sum_{i=0}^{n-1} \int_{M} \frac{i+1}{n+1} \sqrt{-1} \partial \varphi \wedge \bar{\partial} \varphi \wedge \omega^{i} \wedge \omega_{\varphi}^{n-1-i} .
\end{aligned}
$$

From [Aubin 1998] we know that $I$ and $J$ are both semipositive functionals and satisfy

$$
0 \leq I\left(\omega, \omega_{\varphi}\right) \leq(n+1)\left(I\left(\omega, \omega_{\varphi}\right)-J\left(\omega, \omega_{\varphi}\right)\right) \leq n I\left(\omega, \omega_{\varphi}\right) \quad \text { for } \varphi \in \mathscr{H} .
$$

Fix $\varphi \in \mathscr{H}_{0}$. Consider the functional

$$
\Psi(\sigma)=(I-J)\left(\omega_{\varphi}, \sigma^{*} \omega\right)=(I-J)\left(\omega_{\varphi}, \omega_{\rho}\right),
$$

which is defined for any $\sigma$ in the reductive subgroup $\operatorname{Aut}(M)$ with $\sigma^{*} \omega=\omega+\partial \bar{\partial} \rho$. Since $\omega_{\rho}$ is a Kähler-Einstein metric, it satisfies

$$
\log \frac{\omega_{\rho}^{n}}{\omega^{n}}+\rho=0 \quad \text { and } \quad I(\rho)=0 .
$$


If $\omega_{\rho}$ is the minimal point of $\Psi$, for any $u \in \Lambda_{1}\left(\omega_{\rho}\right)$, we have

$$
\int_{M}(\rho-\varphi) u \omega_{\rho}^{n}=0
$$

It is known that $\eta(M) \cong \Lambda_{1}(\omega)$ for any Kähler-Einstein metric $\omega$ [Matsushima 1957]. To prove that the minimizer of $\Psi$ is always attained, it suffices to prove:

Proposition 7.1 [Bando and Mabuchi 1987]. For all

$$
\rho \in\left\{\rho \mid \sigma^{*} \omega=\omega_{\rho}, \sigma \in \operatorname{Aut}_{r}(M), \Psi(\sigma) \leq r\right\},
$$

we have

$$
|\varphi-\rho|_{C^{2, \alpha}\left(g_{\varphi}\right)} \leq C\left(|\varphi|_{C^{4, \alpha}}\right)
$$

Proof. Clearly,

$$
-\triangle_{\varphi}(\rho-\varphi)<n \text { and }-\triangle_{\rho}(\rho-\varphi)>-n .
$$

A lower bound for the Green function is given by

$$
G_{\varphi} \geq-\gamma \frac{D_{\varphi}^{2}}{\operatorname{Vol}_{\varphi}} \doteq-A_{\varphi},
$$

since the volume is constant in a fixed Kähler class and the diameter of $g_{\varphi}$ is bounded by $C \operatorname{diam}(g)$ when $|\varphi|_{C^{2}} \leq C$. Using Green's formula and (7-4), we obtain

(7-5) $\sup _{M}(\rho-\varphi)$

$$
\begin{aligned}
& =\frac{1}{V} \int_{M}(\rho-\varphi) \omega_{\varphi}^{n}-\frac{1}{V} \int_{M} \triangle_{\varphi}(\rho-\varphi)(y)\left(G_{\varphi}(x, y)+A_{\varphi}\right) \omega_{\varphi}^{n}(y) \\
& \leq \frac{1}{V} \int_{M}(\rho-\varphi) \omega_{\varphi}^{n}+n A_{\varphi} .
\end{aligned}
$$

Similarly, we deduce that

(7-6) $\inf _{M}(\rho-\varphi)$

$$
\begin{aligned}
& =\frac{1}{V} \int_{M}(\rho-\varphi) \omega_{\rho}^{n}-\frac{1}{V} \int_{M} \triangle_{\rho}(\rho-\varphi)(y)\left(G_{\rho}(x, y)+A_{\rho}\right) \omega_{\rho}^{n}(y) \\
& \geq \frac{1}{V} \int_{M}(\rho-\varphi) \omega_{\rho}^{n}-n A_{\rho} .
\end{aligned}
$$

Because Ric $(\rho)=\omega_{\rho}$, we have $\operatorname{diam}\left(g_{\rho}\right) \leq \sqrt{2 n-1} \pi$ Myers' theorem. Combining (7-5) and (7-6) we get

$$
\operatorname{Osc}_{M}(\rho-\varphi) \geq \frac{1}{V} \int_{M}(\rho-\varphi)\left(\omega_{\varphi}^{n}-\omega_{\rho}^{n}\right)+C\left(|\varphi|_{C^{2}}\right)
$$


From (7-1) we obtain

$$
\frac{1}{V} \int_{M}(\rho-\varphi)\left(\omega_{\varphi}^{n}-\omega_{\rho}^{n}\right)=I\left(\omega_{\varphi}, \omega_{\rho}\right) \leq(n+1)(I-J)\left(\omega_{\varphi}, \omega_{\rho}\right) \leq(n+1) r .
$$

Since $\omega_{\rho}$ is a Kähler-Einstein metric, we have

$$
\left(\omega_{\varphi}+\sqrt{-1} \partial \bar{\partial}(\rho-\varphi)\right)^{n}=e^{-(\rho-\varphi)+h_{\varphi}} \omega_{\varphi}^{n},
$$

with

$$
\sqrt{-1} \partial \bar{\partial} h_{\varphi}=\operatorname{Ric}\left(\omega_{\varphi}\right)-\omega_{\varphi} \quad \text { and } \quad \int_{M} e^{h_{\varphi}} \omega_{\varphi}^{n}=\operatorname{Vol}(M) .
$$

By using the second-order estimate in [Yau 1978], we get

$$
\begin{aligned}
n+\triangle_{\varphi}(\rho-\varphi) & \leq e^{C \operatorname{Osc}_{M}(\rho-\varphi)} C\left(\sup _{M}\left(\inf _{i \neq k}\left|R_{\varphi i \bar{i} k \bar{k}}\right|\right), \inf _{M} S_{\varphi}, \sup _{M} h_{\varphi}\right) \\
& \leq e^{C \operatorname{Osc}_{M}(\rho-\varphi)} C\left(|\varphi|_{C^{4}}\right) .
\end{aligned}
$$

Then the Krylov estimate shows that $\rho-\varphi$ has $C^{2, \alpha}$ bound.

Thus we also obtain a uniform bound for the gauge $\rho$. Our previous discussion implies:

Corollary 7.2. If $|\varphi|_{C^{4, \alpha}}$ is bounded and $\rho$ is the minimizer of $\Psi$, then $|\varphi-\rho|_{C^{2, \alpha}}$ and $|\rho|_{C^{2, \alpha}}$ are both bounded.

This implies that $g_{\rho}$ is equivalent to $g$.

We now turn to the uniqueness of the critical points of the functional $\Psi$ when $\varphi$ is small. The second variation of $\Psi$ at $\rho$ is given by

$$
D^{2} \Psi_{\rho}(u, v)=\frac{1}{V} \int_{M}\left(1+\frac{1}{2} \triangle_{\rho} \rho\right) u v \omega_{\rho}^{n} .
$$

Lemma 7.3. For all $|\varphi|_{C^{2, \alpha}} \leq \epsilon_{1}$ and $u \in \Lambda_{1}\left(\omega_{\rho}\right)$, the bilinear form $D^{2} \Psi_{\rho}(u, u)$ is positive definite. Hence $\rho$ is unique.

Proof. Note that (7-8) can be rewritten as

$$
\left(\omega_{\rho}+\sqrt{-1} \partial \bar{\partial}(\varphi-\rho)\right)^{n}=e^{-(\varphi-\rho)-h_{\varphi}} \omega_{\rho}^{n} .
$$

By definition, $h_{\varphi}$ is given by

$$
h_{\varphi}=-\log \frac{\omega_{\varphi}^{n}}{\omega^{n}}-\varphi-\log \left(\frac{1}{V} \int_{M} e^{-\varphi}\right) \omega^{n} .
$$

We conclude that

$$
\left|h_{\varphi}\right|_{C^{2, \alpha}\left(g_{\rho}\right)} \leq C \epsilon_{1} \leq \delta,
$$


by the assumption on $\varphi$. Let

$$
C_{\perp}^{2, \alpha}(M)=\left\{\varphi \in C^{2, \alpha}(M) \mid \int_{M} \varphi u \omega_{\rho}^{n} \text { for all } u \in \Lambda_{1}\left(\omega_{\rho}\right)\right\} .
$$

Define the operator of (7-10) by

$$
\Phi(a, b)=\log \frac{\left(\omega_{\rho}+\sqrt{-1} \partial \bar{\partial} a\right)^{n}}{\omega_{\rho}^{n}}+a+b, \quad C_{\perp}^{2, \alpha}(M) \times C^{\alpha}(M) \rightarrow C^{\alpha}(M) .
$$

It is clear from (7-10) that $\Phi\left(\varphi-\rho, h_{\varphi}\right)=0$. The linearized operator of (7-10) at $(a, b)=(0,0)$ is given by

$$
\delta_{a} \Phi(v)=\triangle_{\rho} v+v .
$$

We infer that $\delta_{a} \Phi$ is invertible from $C_{\perp}^{2, \alpha}(M)$ to $C_{\perp}^{\alpha}(M)$. The implicit function theorem implies that there is a small $\delta$ neighborhood of 0 in $C^{\alpha}(M)$ such that when $\left|h_{\varphi}\right|_{C^{2, \alpha}\left(g_{\rho}\right)} \leq \delta$, we have from (7-3) that

$$
|\varphi-\rho|_{C^{2, \alpha}\left(g_{\rho}\right)} \leq C \delta .
$$

Then we use Corollary 7.2 and (7-11) to obtain

$$
|\rho|_{C^{2, \alpha}} \leq|\varphi-\rho|_{C^{2, \alpha}}+|\varphi|_{C^{2, \alpha}} \leq C \epsilon_{1} .
$$

Hence $|\rho|_{C^{2, \alpha}}$ is small if we choose appropriate $\epsilon_{1}$ and the bilinear form $D^{2} \Psi_{\rho}(u, u)$ is positive definite.

\section{Acknowledgements}

The author is grateful to Professor Xiuxiong Chen, who introduced him to this problem and to Kähler geometry. He is also grateful to Professor Weiyue Ding for his constant encouragement and support. He also thanks Professor Xiaohua Zhu for his interest in this problem and many helpful discussions.

\section{References}

[Aubin 1998] T. Aubin, Some nonlinear problems in Riemannian geometry, Springer, Berlin, 1998. MR 99i:58001 Zbl 0896.53003

[Bando 1987] S. Bando, "The $K$-energy map, almost Einstein Kähler metrics and an inequality of the Miyaoka-Yau type”, Tohoku Math. J. (2) 39:2 (1987), 231-235. MR 88k:53092

[Bando and Mabuchi 1987] S. Bando and T. Mabuchi, "Uniqueness of Einstein Kähler metrics modulo connected group actions", pp. 11-40 in Algebraic geometry (Sendai, 1985), edited by T. Oda, Adv. Stud. Pure Math. 10, North-Holland, Amsterdam, 1987. MR 89c:53029 Zbl 0641.53065

[Calabi 1985] E. Calabi, "Extremal Kähler metrics, II", pp. 95-114 in Differential geometry and complex analysis, edited by I. Chavel and H. E. Rauch, Springer, Berlin, 1985. MR 86h:53067 Zbl 0574.58006 
[Calabi and Chen 2002] E. Calabi and X. X. Chen, "The space of Kähler metrics, II", J. Differential Geom. 61:2 (2002), 173-193. MR 2004i:32039 Zbl 1067.58010

[Cao 1985] H. D. Cao, "Deformation of Kähler metrics to Kähler-Einstein metrics on compact Kähler manifolds”, Invent. Math. 81:2 (1985), 359-372. MR 87d:58051 Zbl 0574.53042

[Chen 2000a] X. Chen, "On the lower bound of the Mabuchi energy and its application", Internat. Math. Res. Notices 2000:12 (2000), 607-623. MR 2001f:32042 Zbl 0980.58007

[Chen 2000b] X. Chen, “The space of Kähler metrics”, J. Differential Geom. 56:2 (2000), 189-234. MR 2003b:32031 Zbl 1041.58003

[Chen and Ding 2007] X. Chen and W. Ding, "Ricci flow on surfaces with degenerate initial metrics”, J. Partial Differential Equations 20:3 (2007), 193-202. MR 2008g:53081 Zbl 1174.53025

[Chen and Li 2009] X. Chen and H. Li, "Stability of Kähler-Ricci Flow", J. Geom. Anal. 20:2 (2009), 306-334. Zbl 1185.53074

[Chen and Tian 2002] X. X. Chen and G. Tian, "Ricci flow on Kähler-Einstein surfaces", Invent. Math. 147:3 (2002), 487-544. MR 2003c:53095 Zbl 1047.53043

[Chen and Tian 2006] X. X. Chen and G. Tian, "Ricci flow on Kähler-Einstein manifolds", Duke Math. J. 131:1 (2006), 17-73. MR 2007c:53083 Zbl 1097.53045

[Chen and Tian 2008] X. X. Chen and G. Tian, "Geometry of Kähler metrics and foliations by holomorphic discs", Publ. Math. Inst. Hautes Études Sci. 107 (2008), 1-107. MR 2009g:32048 Zbl 1182.32009

[Chen and Wang 2010] X. Chen and B. Wang, "Remarks on Kähler Ricci flow", J. Geom. Anal. 20:2 (2010), 335-353. MR 2011b:32036 Zbl 1185.53075

[Chen and Zheng 2010] X. Chen and K. Zheng, "The pseudo-Calabi flow", preprint, 2010. to appear in J. Reine Angew. Math. arXiv 1004.2663

[Chen et al. 2008] X. X. Chen, G. Tian, and Z. Zhang, "On the weak Kähler-Ricci flow", preprint, 2008. arXiv 0802.0809

[Donaldson 1999] S. K. Donaldson, "Symmetric spaces, Kähler geometry and Hamiltonian dynamics", pp. 13-33 in Northern California Symplectic Geometry Seminar, edited by Y. Eliashberg et al., Amer. Math. Soc. Transl. Ser. 2 196, Amer. Math. Soc., Providence, RI, 1999. MR 2002b:58008 Zbl 0972.53025

[Donaldson 2004] S. K. Donaldson, "Conjectures in Kähler geometry", pp. 71-78 in Strings and geometry, edited by M. Douglas et al., Clay Math. Proc. 3, Amer. Math. Soc., Providence, RI, 2004. MR 2005i:32024 Zbl 1161.32010

[Fujiki 1978] A. Fujiki, "On automorphism groups of compact Kähler manifolds", Invent. Math. 44:3 (1978), 225-258. MR 58 \#1285 Zbl 0367.32004

[Futaki and Mabuchi 1995] A. Futaki and T. Mabuchi, "Bilinear forms and extremal Kähler vector fields associated with Kähler classes", Math. Ann. 301:2 (1995), 199-210. MR 95m:32039 Zbl 0831.53042

[Hamilton 1982] R. S. Hamilton, "Three-manifolds with positive Ricci curvature", J. Differential Geom. 17:2 (1982), 255-306. MR 84a:53050 Zbl 0504.53034

[Huang and Zheng 2010] H. Huang and K. Zheng, "Stability of Calabi flow near extremal metric", preprint, 2010. to appear in Ann. Scuola Norm. Sup. Pisa Cl. Sci. arXiv 1007.4571

[Kobayashi 1961] S. Kobayashi, "On compact Kähler manifolds with positive definite Ricci tensor", Ann. of Math. (2) 74 (1961), 570-574. MR 24 \#A2922 Zbl 0107.16002 
[Mabuchi 1986] T. Mabuchi, "K-energy maps integrating Futaki invariants", Tohoku Math. J. (2) 38:4 (1986), 575-593. MR 88b:53060 Zbl 0619.53040

[Mabuchi 1987] T. Mabuchi, "Some symplectic geometry on compact Kähler manifolds. I", Osaka J. Math. 24:2 (1987), 227-252. MR 88m:53126 Zbl 0645.53038

[Matsushima 1957] Y. Matsushima, "Sur la structure du groupe d'homéomorphismes analytiques d'une certaine variété kählérienne”, Nagoya Math. J. 11 (1957), 145-150. MR 20 \#995 ZBL 0091.34803

[Munteanu 2009] O. Munteanu, "A sharp estimate for the bottom of the spectrum of the Laplacian on Kähler manifolds”, J. Differential Geom. 83:1 (2009), 163-187. MR 2010j:58071 Zbl 1183.53068

[Pali 2009] N. Pali, "On the solutions of the Aubin equation and the K-energy of Einstein-Fano manifolds", Complex Var. Elliptic Equ. 54:11 (2009), 1019-1054. MR 2011c:32039 Zbl 1181.53038

[Perelman 2002] G. Perelman, "The entropy formula for the Ricci flow and its geometric applications", preprint, 2002. Zbl 1130.53001 arXiv 0211159v1

[Phong and Sturm 2006] D. H. Phong and J. Sturm, "On stability and the convergence of the KählerRicci flow”, J. Differential Geom. 72:1 (2006), 149-168. MR 2007a:53128 Zbl 1125.53048

[Phong et al. 2008] D. H. Phong, J. Song, J. Sturm, and B. Weinkove, "The Kähler-Ricci flow with positive bisectional curvature”, Invent. Math. 173:3 (2008), 651-665. MR 2009f:53103 ZBL 1145. 53050

[Phong et al. 2009] D. H. Phong, J. Song, J. Sturm, and B. Weinkove, "The Kähler-Ricci flow and the $\bar{\partial}$ operator on vector fields”, J. Differential Geom. 81:3 (2009), 631-647. MR 2010b:32035 Zbl 1162.32014

[Phong et al. 2011] D. H. Phong, J. Song, J. Sturm, and B. Weinkove, "On the convergence of the modified Kähler-Ricci flow and solitons”, Comment. Math. Helv. 86:1 (2011), 91-112.

[Rubinstein 2009] Y. A. Rubinstein, "On the construction of Nadel multiplier ideal sheaves and the limiting behavior of the Ricci flow", Trans. Amer. Math. Soc. 361:11 (2009), 5839-5850. MR 2010i:32021 Zbl 1185.53077

[Semmes 1992] S. Semmes, "Complex Monge-Ampère and symplectic manifolds", Amer. J. Math. 114:3 (1992), 495-550. MR 94h:32022 Zbl 0790.32017

[Sesum and Tian 2008] N. Sesum and G. Tian, "Bounding scalar curvature and diameter along the Kähler Ricci flow (after Perelman)”, J. Inst. Math. Jussieu 7:3 (2008), 575-587. MR 2009c:53092

[Simon 1983] L. Simon, "Asymptotics for a class of nonlinear evolution equations, with applications to geometric problems", Ann. of Math. (2) 118:3 (1983), 525-571. MR 85b:58121

[Song and Tian 2009] J. Song and G. Tian, “The Kähler-Ricci flow through singularities”, preprint, 2009. arXiv 0909.4898

[Székelyhidi 2010] G. Székelyhidi, "The Kähler-Ricci flow and K-polystability", Amer. J. Math. 132:4 (2010), 1077-1090. MR 2663648 Zbl 1206.53075

[Tian 2000] G. Tian, Canonical metrics in Kähler geometry, Birkhäuser, Basel, 2000. Notes taken by Meike Akveld. MR 2001j:32024 Zbl 0978.53002

[Tian and Zhu 2000] G. Tian and X. Zhu, "Uniqueness of Kähler-Ricci solitons", Acta Math. 184:2 (2000), 271-305. MR 2001h:32040 Zbl 1036.53052

[Tian and Zhu 2002] G. Tian and X. Zhu, "A new holomorphic invariant and uniqueness of KählerRicci solitons", Comment. Math. Helv. 77:2 (2002), 297-325. MR 2003i:32042 Zbl 1036.53053

[Tian and Zhu 2007] G. Tian and X. Zhu, "Convergence of Kähler-Ricci flow", J. Amer. Math. Soc. 20:3 (2007), 675-699. MR 2007k:53107 Zbl 1185.53078 
[Tian and Zhu 2008] G. Tian and X. Zhu, "Perelman's W-functional and stability of Kähler-Ricci flow”, preprint, 2008. arXiv 0801.3504

[Tosatti 2010] V. Tosatti, "Kähler-Ricci flow on stable Fano manifolds", J. Reine Angew. Math. 640 (2010), 67-84. MR 2011e:32030 Zbl 1189.53069

[Yau 1978] S. T. Yau, "On the Ricci curvature of a compact Kähler manifold and the complex Monge-Ampère equation. I”, Comm. Pure Appl. Math. 31:3 (1978), 339-411. MR 81d:53045 Zbl 0369.53059

[Zhu 2009] X. Zhu, "Stability on Kähler-Ricci flow, I", preprint, 2009. arXiv 0908.1488

Received May 23, 2010. Revised November 18, 2010.

KAI ZHENG

ACADEMy of Mathematics And Systems Science

CHINESE ACADEMY OF SCIENCES

BEIJING, 100190

CHINA

kaizheng@amss.ac.cn 


\title{
PACIFIC JOURNAL OF MATHEMATICS
}

\author{
http://www.pjmath.org \\ Founded in 1951 by
}

E. F. Beckenbach (1906-1982) and F. Wolf (1904-1989)

\section{EDITORS}

V. S. Varadarajan (Managing Editor)

Department of Mathematics

University of California

Los Angeles, CA 90095-1555

pacific@math.ucla.edu

Vyjayanthi Chari

Department of Mathematics

University of California

Riverside, CA 92521-0135

chari@math.ucr.edu

\section{Robert Finn}

Department of Mathematics Stanford University

Stanford, CA 94305-2125

finn@math.stanford.edu

Kefeng Liu

Department of Mathematics

University of California

Los Angeles, CA 90095-1555

liu@math.ucla.edu
Darren Long

Department of Mathematics

University of California

Santa Barbara, CA 93106-3080

long@math.ucsb.edu

Jiang-Hua Lu

Department of Mathematics

The University of Hong Kong

Pokfulam Rd., Hong Kong jhlu@maths.hku.hk

Alexander Merkurjev

Department of Mathematics University of California

Los Angeles, CA 90095-1555 merkurev@math.ucla.edu
Sorin Popa

Department of Mathematics

University of California

Los Angeles, CA 90095-1555

popa@math.ucla.edu

Jie Qing

Department of Mathematics

University of California

Santa Cruz, CA 95064

qing@ cats.ucsc.edu

Jonathan Rogawski

Department of Mathematics

University of California

Los Angeles, CA 90095-1555

jonr@math.ucla.edu

\section{PRODUCTION}

pacific@math.berkeley.edu

Silvio Levy, Scientific Editor Matthew Cargo, Senior Production Editor

ACADEMIA SINICA, TAIPEI

CALIFORNIA INST. OF TECHNOLOGY

INST. DE MATEMÁTICA PURA E APLICADA

KEIO UNIVERSITY

MATH. SCIENCES RESEARCH INSTITUTE

NEW MEXICO STATE UNIV.

OREGON STATE UNIV.

\section{SUPPORTING INSTITUTIONS}

STANFORD UNIVERSITY
UNIV. OF BRITISH COLUMBIA
UNIV. OF CALIFORNIA, BERKELEY
UNIV. OF CALIFORNIA, DAVIS
UNIV. OF CALIFORNIA, LOS ANGELES
UNIV. OF CALIFORNIA, RIVERSIDE
UNIV. OF CALIFORNIA, SAN DIEGO
UNIV. OF CALIF., SANTA BARBARA

UNIV. OF CALIF., SANTA CRUZ

UNIV. OF MONTANA

UNIV. OF OREGON

UNIV. OF SOUTHERN CALIFORNIA

UNIV. OF UTAH

UNIV. OF WASHINGTON

WASHINGTON STATE UNIVERSITY

These supporting institutions contribute to the cost of publication of this Journal, but they are not owners or publishers and have no responsibility for its contents or policies.

See inside back cover or www.pjmath.org for submission instructions.

The subscription price for 2011 is US \$420/year for the electronic version, and \$485/year for print and electronic.

Subscriptions, requests for back issues from the last three years and changes of subscribers address should be sent to Pacific Journal of Mathematics, P.O. Box 4163, Berkeley, CA 94704-0163, U.S.A. Prior back issues are obtainable from Periodicals Service Company, 11 Main Street, Germantown, NY 12526-5635. The Pacific Journal of Mathematics is indexed by Mathematical Reviews, Zentralblatt MATH, PASCAL CNRS Index, Referativnyi Zhurnal, Current Mathematical Publications and the Science Citation Index.

The Pacific Journal of Mathematics (ISSN 0030-8730) at the University of California, c/o Department of Mathematics, 969 Evans Hall, Berkeley, CA 94720-3840, is published monthly except July and August. Periodical rate postage paid at Berkeley, CA 94704, and additional mailing offices. POSTMASTER: send address changes to Pacific Journal of Mathematics, P.O. Box 4163, Berkeley, CA 94704-0163.

PJM peer review and production are managed by EditFLOW ${ }^{\mathrm{TM}}$ from Mathematical Sciences Publishers.

PUBLISHED BY PACIFIC JOURNAL OF MATHEMATICS

at the University of California, Berkeley 94720-3840

A NON-PROFIT CORPORATION

Typeset in IATEX

Copyright $(2011$ by Pacific Journal of Mathematics 


\section{PACIFIC JOURNAL OF MATHEMATICS}

Volume $251 \quad$ No. $2 \quad$ June 2011

Two Kazdan-Warner-type identities for the renormalized volume coefficients and the Gauss-Bonnet curvatures of a Riemannian metric

BIN GUO, ZHENG-CHAO HAN and HAIZHONG LI

Gonality of a general ACM curve in $\mathbb{P}^{3}$

ROBIN HARTSHORNE and ENRICO SCHLESINGER

Universal inequalities for the eigenvalues of the biharmonic operator on submanifolds

SAÏD ILIAS and OLA MAKHOUL

Multigraded Fujita approximation

SHIN-YaO Jow

Some Dirichlet problems arising from conformal geometry

QI-RUi Li and WEIMIN SHENG

Polycyclic quasiconformal mapping class subgroups

KATSUHIKO MATSUZAKI

On zero-divisor graphs of Boolean rings

ALI MOHAMMADIAN

Rational certificates of positivity on compact semialgebraic sets

VICTORIA POWERS

Quiver grassmannians, quiver varieties and the preprojective algebra

Alistair Savage and Peter Tingley

Nonautonomous second order Hamiltonian systems

\section{MARTIN SCHECHTER}

Generic fundamental polygons for Fuchsian groups

AKIRA USHIJIMA

Stability of the Kähler-Ricci flow in the space of Kähler metrics

KAI ZHENG

The second variation of the Ricci expander entropy 